\title{
Genome-wide identification and comparative analysis of diacylglycerol kinase $(D G K)$ gene family and their expression profiling in Brassica napus under abiotic stress
}

Fang Tang ${ }^{1,2}$, Zhongchun Xiao ${ }^{1,2}$, Fujun Sun ${ }^{1,2}$, Shulin Shen ${ }^{1,2}$, Si Chen ${ }^{1,2}$, Rui Chen ${ }^{1,2}$, Meichen Zhu ${ }^{1,2}$, Qianwei Zhang ${ }^{1,2}$, Hai Du ${ }^{1,2}$, Kun Lu ${ }^{1,2}$, Jiana $\mathrm{Li}^{1,2^{*}}$ and Cunmin Qu ${ }^{1,2^{*}}$ (D)

\begin{abstract}
Background: Diacylglycerol kinases (DGKS) are signaling enzymes that play pivotal roles in response to abiotic and biotic stresses by phosphorylating diacylglycerol (DAG) to form phosphatidic acid (PA). However, no comprehensive analysis of the DGK gene family had previously been reported in B. napus and its diploid progenitors (B. rapa and B. oleracea).

Results: In present study, we identified 21, 10, and 11 DGK genes from B. napus, B. rapa, and B. oleracea, respectively, which all contained conserved catalytic domain and were further divided into three clusters. Molecular evolutionary analysis showed that speciation and whole-genome triplication (WGT) was critical for the divergence of duplicated DGK genes. RNA-seq transcriptome data revealed that, with the exception of BnaDGK4 and BnaDGK6, BnaDGK genes have divergent expression patterns in most tissues. Furthermore, some DGK genes were upregulated or downregulated in response to hormone treatment and metal ion (arsenic and cadmium) stress. Quantitative realtime PCR analysis revealed that different BnaDGK genes contribute to seed oil content.
\end{abstract}

Conclusions: Together, our results indicate that DGK genes have diverse roles in plant growth and development, hormone response, and metal ion stress, and in determining seed oil content, and lay a foundation for further elucidating the roles of DGKs in Brassica species.

Keywords: Brassica, Diacylglycerol kinase, Gene family, Phylogenetic analysis, Expression profile

\footnotetext{
*Correspondence: ljn1950@swu.edu.cn; drqucunmin@swu.edu.cn

'Chongqing Engineering Research Center for Rapeseed, College of

Agronomy and Biotechnology, Southwest University, Chongqing 400716,

China

Full list of author information is available at the end of the article
}

(c) The Author(s). 2020 Open Access This article is licensed under a Creative Commons Attribution 4.0 International License, which permits use, sharing, adaptation, distribution and reproduction in any medium or format, as long as you give appropriate credit to the original author(s) and the source, provide a link to the Creative Commons licence, and indicate if changes were made. The images or other third party material in this article are included in the article's Creative Commons licence, unless indicated otherwise in a credit line to the material. If material is not included in the article's Creative Commons licence and your intended use is not permitted by statutory regulation or exceeds the permitted use, you will need to obtain permission directly from the copyright holder. To view a copy of this licence, visit http://creativecommons.org/licenses/by/4.0/ The Creative Commons Public Domain Dedication waiver (http://creativecommons.org/publicdomain/zero/1.0/) applies to the data made available in this article, unless otherwise stated in a credit line to the data. 


\section{Background}

In plants, important signaling molecules such as phosphatidylinositol lipids phosphatidic acid (PA), diacylglycerol (DAG), and some lysophospholipids can be activated by distinct environmental stresses, triggering signal transduction cascades and endowing plants with stress resistance $[1,2]$. Among these molecules, PA is a key second messenger in the responses to various stresses. PA levels are typically low, accounting for $0.67 \%$ of total phospholipids, and PA formation depends on the perception of extracellular stimuli [3], such as drought [4], salinity [5], chilling damage [6], osmotic pressure [7], wounding [8], and pathogen attack [9], or of phytohormones such as ethylene [8], abscisic acid (ABA) [10], brassinolide (BR) [11], and gibberellic acid (GA) [12, 13]. Plants' ability to overcome such stress events depends on the role of PA in signal perception and transduction [2, 14, 15]. PA is a precursor to all phosphoglycerolipids and its bioaccumulation is pivotal for lipid metabolic flux and membrane composition [16]. PA is generated in the plasma membrane by two different biosynthetic pathways in eukaryotic cells. In the first pathway, phospholipase D (PLD) hydrolyzes phosphatidylcholine $(\mathrm{PC})$ and phosphatidylethanolamine (PE) to generate PA directly. In the second pathway, phospholipase $\mathrm{C}$ (PLC) hydrolyzes phosphatidylinositol-4,5-bisphosphate to produce DAG, which is in turn phosphorylated by diacylglycerol kinase (DGK) to yield PA [16].

Previous studies have demonstrated that PA interacts with $\mathrm{ABI} 1$ phosphatase $2 \mathrm{C}$, thus promoting ABA signaling in $A$. thaliana [10]. PLD $\alpha$ gene expression, protein levels, and enzymatic activity all increase in $A$. thaliana when the leaves are treated with ethylene [8]. In $B$. napus roots, exogenous application of 24-epibrassinolide (EBL) affects PA synthesis through the PLC/DGK pathway under optimal salinity conditions [17]. Furthermore, $D G K$ genes are rapidly but transiently regulated in different plant tissues in response to beneficial elements and other ions, including silver (Ag), aluminum (Al), arsenic (As), cadmium (Cd), chromium (Cr), mercury $(\mathrm{Hg})$, and sodium (Na) [18]. Notably, DGK genes may be candidates for influencing seed oil content in B. napus, according to recent unpublished data from our laboratory. Additionally, diacylglycerol transferase (DGAT) catalyzes DAG to generate triacylglycerol (TAG), which is a major component of vegetable oils in oilseed crops [19]. Therefore, the relative flux of TAG synthesis from de-novo-synthesized or PC-derived DAG can greatly affect the final seed oil content. The $D G K$ gene family has been widely characterized in the context of plant stress tolerance, including tomato (Solanum lycopersicum, SlDGK) [20], Arabidopsis thaliana (AtDGK) [21], maize (Zea mays, ZmDGK) [22], rice (Oryza sativa, OsDGK) [23], apple (Malus domestica, MdDGK) [4], and soybean (Glycine max, GmDGK) [24]. However, the genomic analysis of the DGK gene family in Brassica species has not been reported.

Brassica napus (AACC, 2n = 38), a typical allotetraploid of the Brassica genus, is an important oil crop planted worldwide, which originated from the hybridization and polyploidization of $B$. oleracea (CC, $2 \mathrm{n}=18$ ) and $B$. rapa (AA, $2 \mathrm{n}=20$ ) [25]. Whole-genome sequences of $B$. rapa, $B$. oleracea, and $B$. napus have recently been assembled [25-27], providing valuable resources for studying the DGK gene family in Brassica species. In this study, we identified DGKs in the Brassica species and investigated their gene structures, conserved domains, protein properties, evolution, and cis-acting elements through bioinformatics analysis. We also evaluated the expression patterns of BnaDGKs in different tissues and their responses to hormone treatment and metal ion induction. Additionally, we studied BnaDGK expression patterns in two B. napus cultivars with different seed oil contents. This genome-wide identification of $D G K$ gene family members in three Brassica species provides strong evidence of functional homologies among these DGK genes in Brassica species.

\section{Results \\ Identification and chromosomal distribution of $D G K$ genes in B. napus, $B$. rapa, and B. oleracea}

We identified 21,10 , and 11 DGK genes in B. napus, $B$. rapa, and $B$. oleracea, respectively. $B$. napus had the same number of $D G K$ genes as $B$. rapa and $B$. oleracea combined, indicating that $D G K$ genes may not have experienced a gene-loss event during polyploidization. In addition, the $B$. napus $D G K$ proteins (BnaDGKs) were between 450 and 720 amino acids (aa) in length, corresponding to coding sequence (CDS) lengths of 1353 to $2163 \mathrm{bp}$; their molecular weights ranged from $49.93 \mathrm{kDa}$ to $79.30 \mathrm{kDa}$; and their pIs from 5.88 to 8.93 (Additional file 8: Table S1).

The number of exons in the $D G K$ genes varied from 7 to 15 , with the maximum in BnaDGK6-2 (Additional file 8: Table S1). Analysis of the physical and chemical characteristics of $21 D G K$ proteins in $B$. rapa and $B$. oleracea revealed that, upon calculation, the average values were approximately equal in $B$. napus and its diploid progenitors.

In plants, previous studies were conducted on the subcellular localization of DGKs to the nucleus, plasma membrane, cytoskeleton, and chloroplast [28, 29]. In present study, the subcellular location of all DGKs was predicted using the PSORT website (Additional file 8: Table S1). Results showed that most members of cluster 1 were located in the endoplasmic reticulum, except BnaDGK1-2, BnaDGK2-1, BolDGK1-2, and BolDGK22 in the nucleus, in accordance with that $A t D G K 1$ and 
AtDGK2 located in endoplasmic reticulum membranes possess amino-terminal hydrophobic segments that are sufficient to target proteins to the cell membrane to play their role in many signal transduction processes [30]; the members of cluster 2 were located in different parts, for example, most of $D G K 3$ and $D G K 7$ were located in the peroxisome and $D G K 4 s$ were localized in the chloroplast, while BnaDGK3-3 and BolDGK3-2 occurred in the mitochondrion; while $D G K 5$ and DGK6 in cluster 3 were predicted to be located in the peroxisome and cytoplasm, respectively (Additional file 8: Table S1). These subcellular localization results were in accordance with the published report [24] and suggested that DGKs have been widely conserved in the same clusters. Furthermore, the diverse localization of plant DGKs implies that they might be actively involved in different cellular processes during development and abiotic stress.

Additionally, we investigated the chromosomal localization of $D G K$ genes in Brassica species based on their physical position in the GFF3 files. In total, 20 of the 21 full-length $D G K s$ of $B$. napus mapped to the assembled 11 chromosomes (9 in An genomes and 11 in Cn genomes), while only one gene was assigned to Ann (random A-genome chromosome). In B. oleracea, 7 of the 11 full-length $D G K s$ were distributed on 5 chromosomes, with the remaining 4 genes randomly located on 4 scaffolds. In $B$. rapa, all full-length $D G K s$ were positioned on 6 of the 10 assembled chromosomes (Additional file 1: Fig. S1). Notably, chromosomes An03, An09, Cn03, Ar03, and Ar09 contained the maximum number of genes, i.e., three, whereas chromosomes $\mathrm{Cn} 02, \mathrm{Cn} 07, \mathrm{Cn} 09, \mathrm{Co} 03$, and $\mathrm{CoO} 7$ each contained two genes. This result suggests that DGKs have uneven distributions in different species. Statistical analysis showed that seven gene pairs have maintained their relative positions between the An subgenome of B. napus and the Ar subgenome of B. rapa, and six gene pairs have maintained their relative positions between the $C n$ subgenome of $B$. napus and the Co genome of B. oleracea (Additional file 1: Fig. S1).

\section{Multiple sequence alignments and sequence characterization of the DGK genes}

To explore the structure of DGK protein sequences, we performed multiple sequence alignments using ClustalX and visualized the results with Jalview and DOG 2.0. The sequences of two diacylglycerol/phorbol ester (DAG/PE)-binding domains (C1 domain, PF00130; Additional file 2: Fig. S2) indicate that the first and second DAG/PE-binding domains harbor the sequences $\mathrm{HX}_{14} \mathrm{CX}_{2} \mathrm{CX}_{19 \sim 22} \mathrm{CX}_{2} \mathrm{CX}_{4} \mathrm{HX}_{2} \mathrm{CX}_{7} \mathrm{C}$ and $\mathrm{HX}_{18} \mathrm{CX}_{2} \mathrm{CX}_{16}$ $\mathrm{CX}_{2} \mathrm{CX}_{4} \mathrm{HX}_{2} \mathrm{CX}_{11} \mathrm{C}$, respectively. The two extremely conserved $\mathrm{C}_{6} / \mathrm{H}_{2}$ cores were also observed in cluster 1 $D G K s$ of other plants, such as soybean [24], apple [4], and maize [22]. Moreover, the alignment revealed that, except for the orthologs of $A t D G K 2$, almost all $D G K s$ possess a presumed ATP-binding motif with a GXGXXG consensus sequence in their catalytic domain (DGKc), where $G$ represents glycine and $X$ represents any other amino acid (Additional file 3: Fig. S3). Furthermore, in the homologs of $A t D G K 2$, glycine $(\mathrm{G})$ is replaced by alanine (A), but this change does not render the enzyme inactive [21]. Interestingly, BnaDGK2-1, with the shortest protein sequence in cluster 1 , lacked the $\mathrm{C}_{6} / \mathrm{H}_{2}$ cores, upstream basic region, and an extended cysteine-rich (extCRD-like) domain; it seems probable that BnaDGK2-1 lost these domains during evolution.

A schematic diagram of the functional domains of cluster 1 DGK proteins drawn using DOG 2.0 showed that compared to the other two clusters, two DAG/PE binding domains $(\mathrm{C} 1)$, the upstream basic region, and a conserved 15-amino-acid extension were specific to cluster 1 (Additional file 4: Fig. S4). In the upstream basic region, YT and VP residues remained as front and back boundaries, respectively, except in homologs of AtDGK1, in which VP was replaced with TP. Furthermore, the second DAG/PE-binding domain included a preserved 15-aa extension, an extCRD-like domain; whereas the combination of the extCRD-like domain and DGKc domain is indispensable to the function of the functionally active DGKs [31]. Overall, the DGKs of cluster 1 in $A$. thaliana, rice, apple, soybean, and $B$. napus, as well as its two diploid progenitors have a universal framework: (YT-upstream basic region-VP/TP) - (3 aa) - (DAG/PEbinding domain 1) - (12 aa) - (DAG/PE-binding domain 1/extCRD-like domain) - ( 130 aa) - (DGKc/ DGKa domain). These results suggest that the structure of $D G K s$ is extremely conserved in cluster 1 .

\section{Phylogenetic tree and syntenic analysis of the DGKs in $B$. napus, B. rapa, and B. oleracea}

To explore the evolutionary relatedness of DGKs between A. thaliana and Brassica, we performed the multiple sequence alignment of DGKs using MUSCLE. Subsequently, we constructed a phylogenetic tree based on the DGK protein sequences, including $7,21,10$, and 11 protein sequences from $A$. thaliana, $B$. napus, $B$. rapa, and $B$. oleracea, respectively (Fig. 1). Generally, the gene number in the $B$. rapa, and $B$. oleracea genome was notably less than three times the $A$. thaliana gene number because some genes might be lost during polyploidy speciation. For example, the $A$. thaliana genes AtDGK4, AtDGK6, and AtDGK7 have at most only two homologs in each of the three Brassica species. By contrast, the remaining AtDGK orthologs possess at least three homologs in B. napus. Additionally, the phylogenetic tree is clearly divided into three clusters, designated clusters 1,2 , and 3 , in accordance with previous studies 


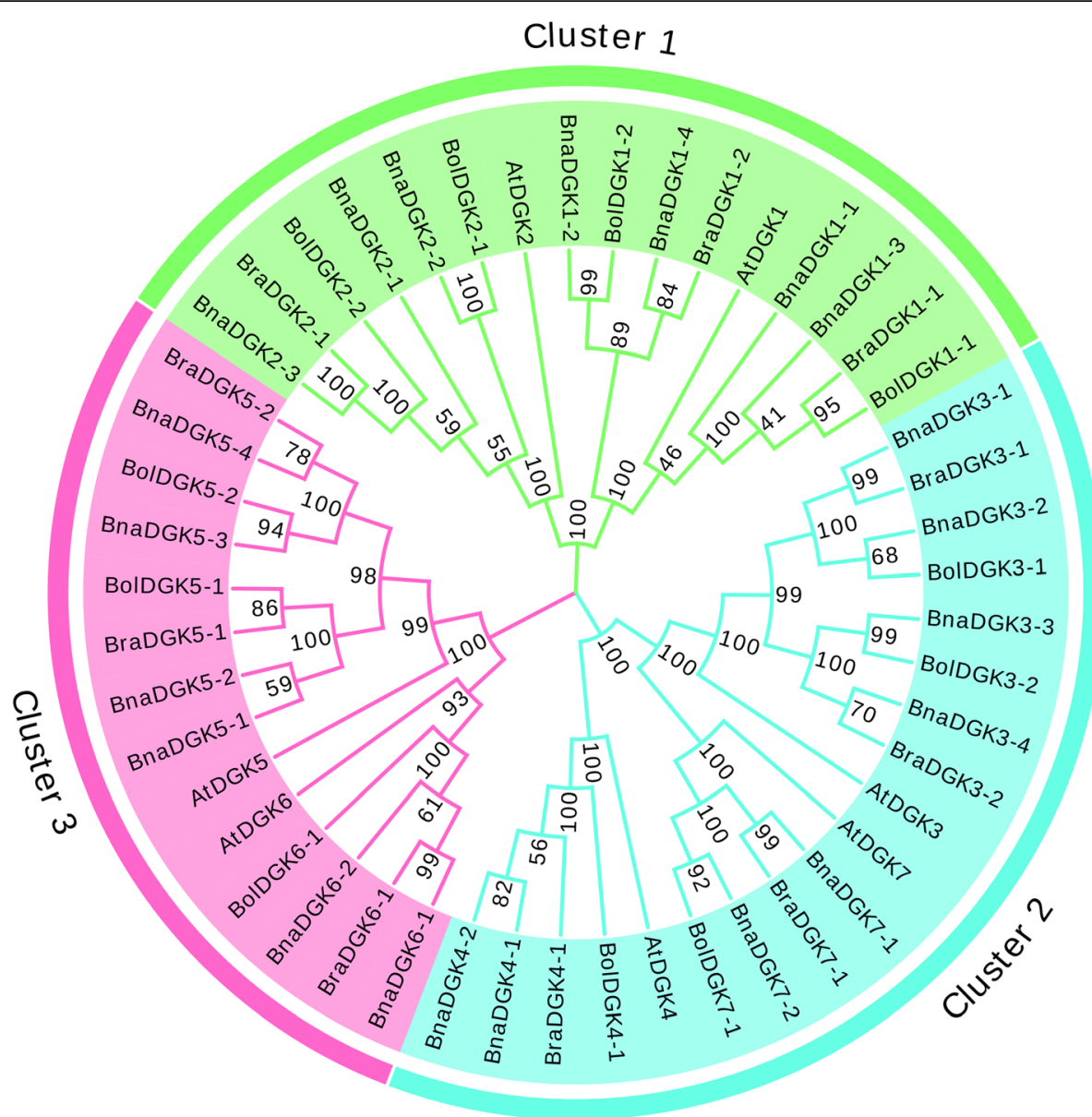

Fig. 1 The phylogenetic tree analysis of DGK proteins between A. thaliana and Brassica species. The MEGA7.0.26 was used to do the phylogenetic analysis with the neighbor joining method

in A. thaliana [21]. Among them, the DGK genes in $B$. napus were closely associated with their corresponding genes in B. oleracea and B. rapa in each clade, suggesting that these genes might have undergone wholegenome duplication events from diploid parental species (B. oleracea and B. rapa) to allotetraploid B. napus, which had similar functions.

We also performed collinearity analysis of DGKs in $A$. thaliana and the three Brassica species to explore the evolutionary relationship of DGKs (Fig. 2). A total of 22 collinear gene pairs between Brassica and A. thaliana were identified, including 9 AT\&Bna gene pairs, 7 AT\&Bra gene pairs, and 6 AT\&Bol gene pairs (Additional file 8: Table S2). Furthermore, these 21 $D G K s$ in B. napus were identified as three homologous gene pairs on homologous chromosomes, while three pairs of genes were not located on homologous chromosomes. For example, the gene pair BnaDGK2-3\& BnaDGK2-1 was distributed on the An06 and $\mathrm{Cn} 03$ chromosomes, respectively, possibly as a result of chromosomal rearrangement and segmental duplication
(Additional file 8: Table S2). Our results suggested that the DGKs might undergo gene duplication or loss from the diploid parental species (B. oleracea and $B$. rapa) to the allotetraploid $B$. napus. Overall, these findings provide the clues for investigating the expansion mechanisms and functional characteristics of $D G K$ family genes in Brassica species.

\section{The expansion of DGK genes in Brassica species}

We also studied the $D G K$ gene duplication types using MCScanX to determine the expansion patterns of the DGK gene family in Brassica species. A total of 101,040 genes annotated in the B. napus genome [25] were examined in this study, and 21,680 genes $(21.46 \%)$ were dispersed and 74,035 genes (73.27\%) might have undergone segmental duplication. Among them, all identified $D G K$ genes belong to segmental duplication except for one dispersed gene BnaDGK4-1 (Additional file 8: Table S1). Moreover, all DGK genes of B. oleracea and B. rapa were derived from segmental duplication. Our findings 


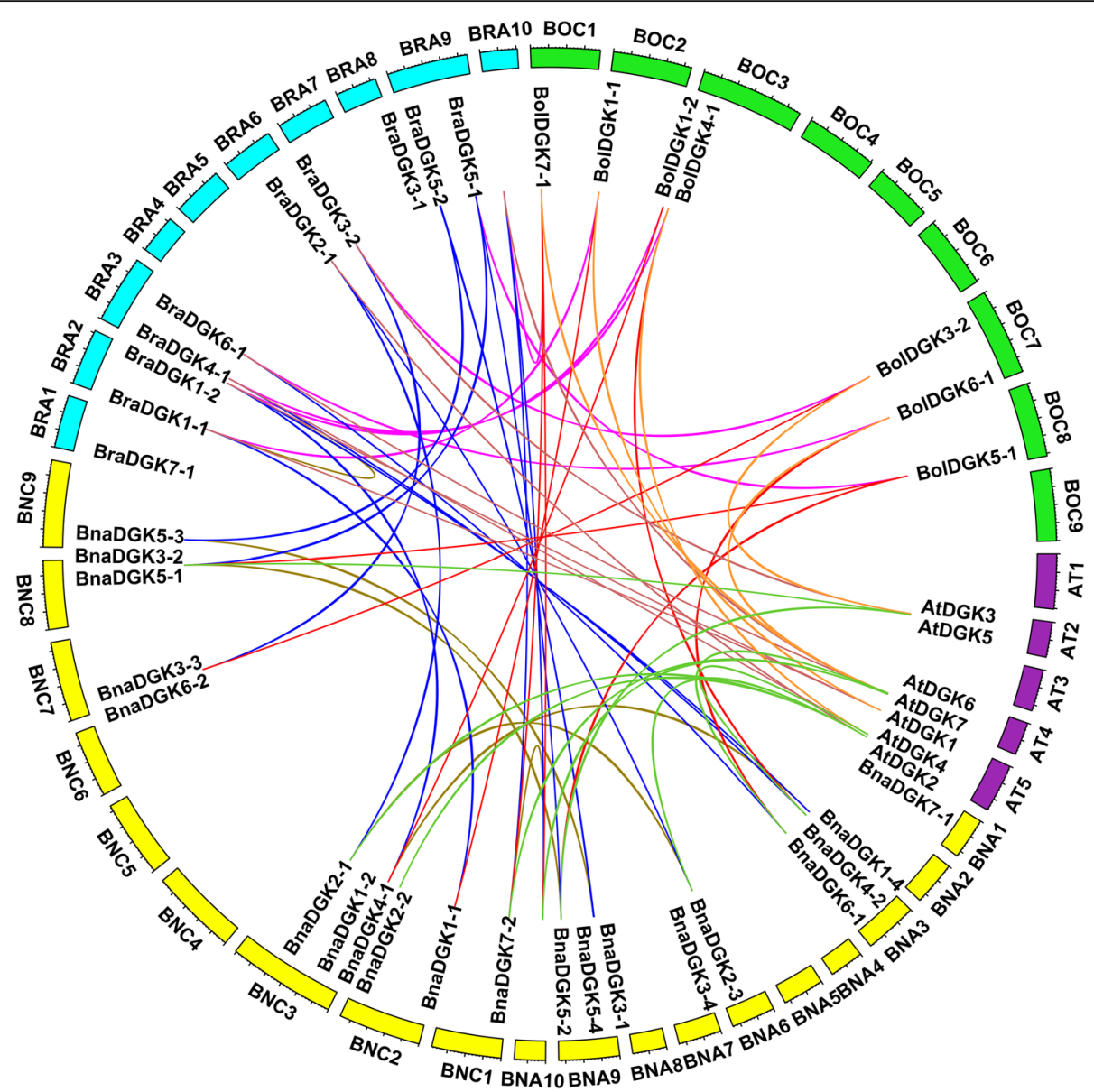

Fig. 2 Genome-wide synteny analysis of DGK genes from B. rapa (Bra), B. oleracea (Bol), B. napus (Bna), and A. thaliana (Atchr). The outer circle indicates the locations of the AtDGKs, BnaDGKs, BraDGKs, and BolDGKs on each chromosome. The inner circle indicates the chromosome number of A. thaliana (Atchr1-5) in purple, B. rapa (BraA1-10) in blue, B. oleracea (BolC1-9) in green, B. napus (BnaA1-10 and BnaC1-9) in yellow. The syntenic DGKs genes were linked using different color lines

showed that segmental duplication appears to play an important role in DGK gene expansion in Brassica species.

The allopolyploid $B$. napus was formed by hybridization of B. rapa and B. oleracea, and its estimated formation time was approximately $\sim 7500$ years ago [25]. To explore the selective pressure on the DGKS after duplication events, we calculated the nonsynonymous $\left(K_{a}\right)$ and synonymous $\left(K_{s}\right)$ substitution rates and the $K_{a} / K_{s}$ ratio for the 69 identified syntenic gene pairs in the three Brassica species and A. thaliana (Additional file 8: Table S2). $K_{a} / K_{s}=1$ signifies that genes have experienced neutral selection, whereas $K_{a} / K_{s}>1$ or $K_{a} / K_{s}<1$ indicate that genes have experienced positive or negative selection, respectively. As a result, the $K_{a} / K_{s}$ values between the Brassica species and A. thaliana ranged from 0.08 to 0.27 . The $K_{a} / K_{s}$ values for most duplicated $D G K$ gene pairs in $B$. napus and each of its diploid progenitors (B. rapa and B. oleracea) were $<1$, except for one gene pair (BnaDGK7-2\&BolDGK7-1) with a value $>1$. Furthermore, for three gene pairs (BnaDGK11\&BolDGK1-1, BnaDGK2-1\&BolDGK2-2, BnaDGK7$1 \& B r a D G K 7-1)$, there were no $K_{a} / K_{s}$ values, because the two genes of each pair had exactly the same CDS. These results showed that most $D G K$ genes have undergone purified selection, whereas the gene pair BnaDGK7-2\&BolDGK7-1 has been positively selected (Additional file 8: Table S2).

In addition, the divergence time of the duplicated genes was estimated by calculating $K_{s}$ values. Our estimation showed that the divergence time ranged from 12.11 to 16.75 million years ago (MYA) and averaged 14.43 MYA between the Brassica species and A. thaliana (Additional file 8: Table S2). This result indicated that the divergence of Brassica DGK genes occurred at 14.43 MYA, in accordance with the WGT event between Brassica species and A. thaliana thought to have occurred approximately 9-15 MYA [32]. For the 6 
paralogous gene pairs in $B$. napus, we used $K_{s}$ values to estimate the time of the whole-genome duplication event. The $K_{s}$ values ranged from 0.07 to 0.17 , with an average of 0.12 . The corresponding derived divergence time varied from 2.48 to 5.83 MYA, with an average of 3.84 MYA, which is considerably lower than the average value in $B$. napus, indicating that the divergence of the $D G K$ genes in B. napus occurred well after the Brassica WGT event (Additional file 8: Table S2). This may be because the formation of the $B$. napus species and the Brassica WGT event had little effect on the divergence of the syntenic $D G K$ genes in B. napus.

\section{Gene structure, domain, and conserved motif analysis}

We studied the intron-exon structures of the $D G K$ genes to determine the structural diversity of $D G K$ genes in different clusters and to explore whether the gene structure changed during polyploidization (Fig. 3). Each $D G K$ cluster possesses a different gene structure. The structure of $D G K$ genes from cluster 1 was significantly more conserved than the other two clusters, with an average of 7 exons. Of 16 DGK genes in cluster 1, 13 gene pairs had an identical gene structure with the corresponding $A$. thaliana homologs, with 7 exons, whereas 3 genes had 9 exons. By comparison, the number of exons varied from 9 to 12 and from 11 to 15 in clusters 2 and 3, respectively. In cluster 2, 12 out of $19 D G K$ genes had 10 exons. In cluster 3, out of $14 D G K$ genes, 6 genes were composed of 11 exons and 5 genes had 12 exons. In addition, we performed a comparative analysis of 17 gene pairs with the closest genetic distance in the phylogenetic tree, which we considered might have a direct evolutionary relationship. Of these 17 gene pairs, 16 gene pairs had an identical gene structure. Therefore, the $D G K$ genes shared a high similarity in exon number during polyploidization.

Furthermore, we analyzed the domain location of DGK proteins to identify the changes in the domain's position between different clusters or different species (Fig. 3). Although, all the DGK proteins assessed contained a catalytic domain (DGKc, PF00781) and an accessory domain (DGKa, PF00609), only cluster 1 had two C1_1 (PF00130) domains. Furthermore, BnaDGK2-1 had no C1_1 domain and had the shortest sequence in cluster 1 . The $\mathrm{C} 1$ (or DAG/PE binding) domain binds an important secondary messenger DAG, as well as the analogous phorbol esters (PE) [33]. Additionally, the positions and lengths of functional domains belonging to the same clusters were generally identical, although they varied between clusters. For instance, most DGKc domains of cluster 1 started from the 261st aa, while the DGKc domains of cluster 2 began at the 82nd aa and the DGKc domains of cluster 3 began at the 35th aa. The location of the DGKa domain was likewise different between the three clusters.

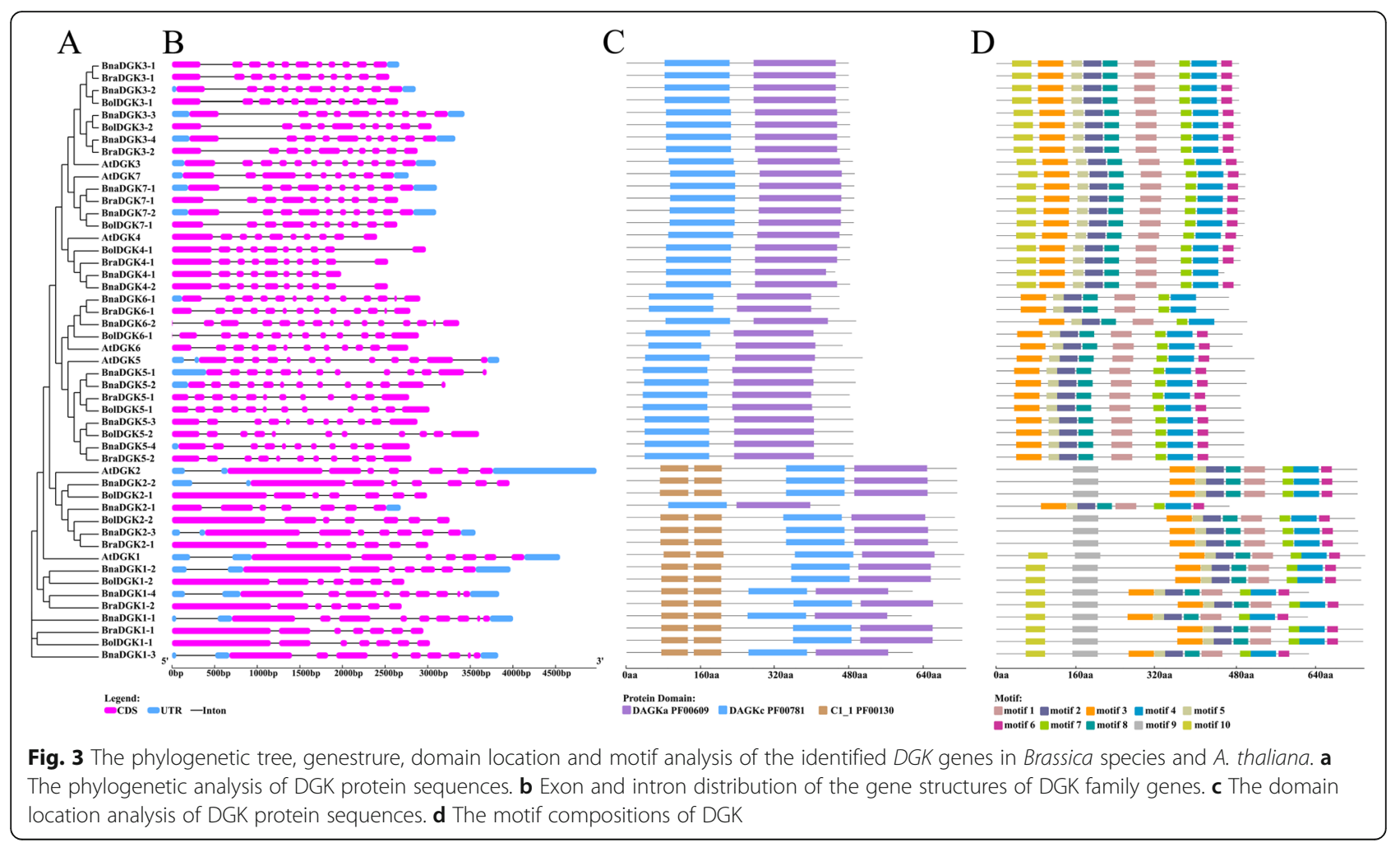


Next, we used the MEME online tool to search for 10 conserved motifs in DGK proteins (Fig. 3 and Additional file 5: Fig. S5). We detected 10 motifs, among which 7 (motifs 1, 2, 3, 4, 5, 7, and 8) occurred in all DGK proteins. In general, DGK proteins in the same cluster displayed parallel motif components. Notably, motif 9 existed only in cluster 1 and constituted the second C1_1 domain of DGKs, while motif 10 existed in 28 DGK proteins, including $19 D G K s$ in cluster 2 as well as the DGKs homologous to AtDGK1. Taken together, $D G K$ genes were highly conserved at the protein level among Brassica species and A. thaliana.

\section{Expression profiles of DGK genes in different tissues of Brassica species}

As we know, the gene functions were predicted by the expression patterns during plant development. To explore the expression patterns of BnaDGKs, we analyzed their expression levels in 12 distinct tissues at different developmental stages, including radicle, hypocotyl, cotyledon, root, stem, young leaf, mature leaf, petal, pistil, stamen, seed, and seed pericarp. All samples of 12 tissues at different developmental stages and time points in B. napus are detailed in Additional file 8: Table S4. Based on the transcriptome sequencing datasets from $B$. napus ZS11 (the BioProject ID PRJNA358784), we found that 7 BnaDGKs were highly expressed in distinct tissues and 7 BnaDGKs were expressed in specific tissues, whereas 3 BnaDGKs had low expression levels (Fig. 4a). The remaining 4 BnaDGKs (BnaDGK4 and BnaDGK6) exhibited almost no expression in most tissues, except that BnaDGK4 was detected in Sta_i and BnaDGK6 was detected in Se_10d (Fig. 4a). Several genes were expressed in all tissues except LeY_f, Sta_i, and seed, including BnaDGK1-1, BnaDGK1-2, BnaDGK1-3, BnaDGK3-3, BnaDGK3-4, BnaDGK5-1, and BnaDGK5-2. Notably, BnaDGK2-2 and BnaDGK2-3 had high expression in Sta_i (Fig. 4a). Our results showed that DGKs had relative expression levels in young tissues, in accordance with the previous results [18]. In addition, some genes displayed the tissue-specific expression profiles, for example, BnaDGK1-4 in stem, BnaDGK7-1 in leaves and silique pericarps, and $B n a D G K 7-2$ in silique

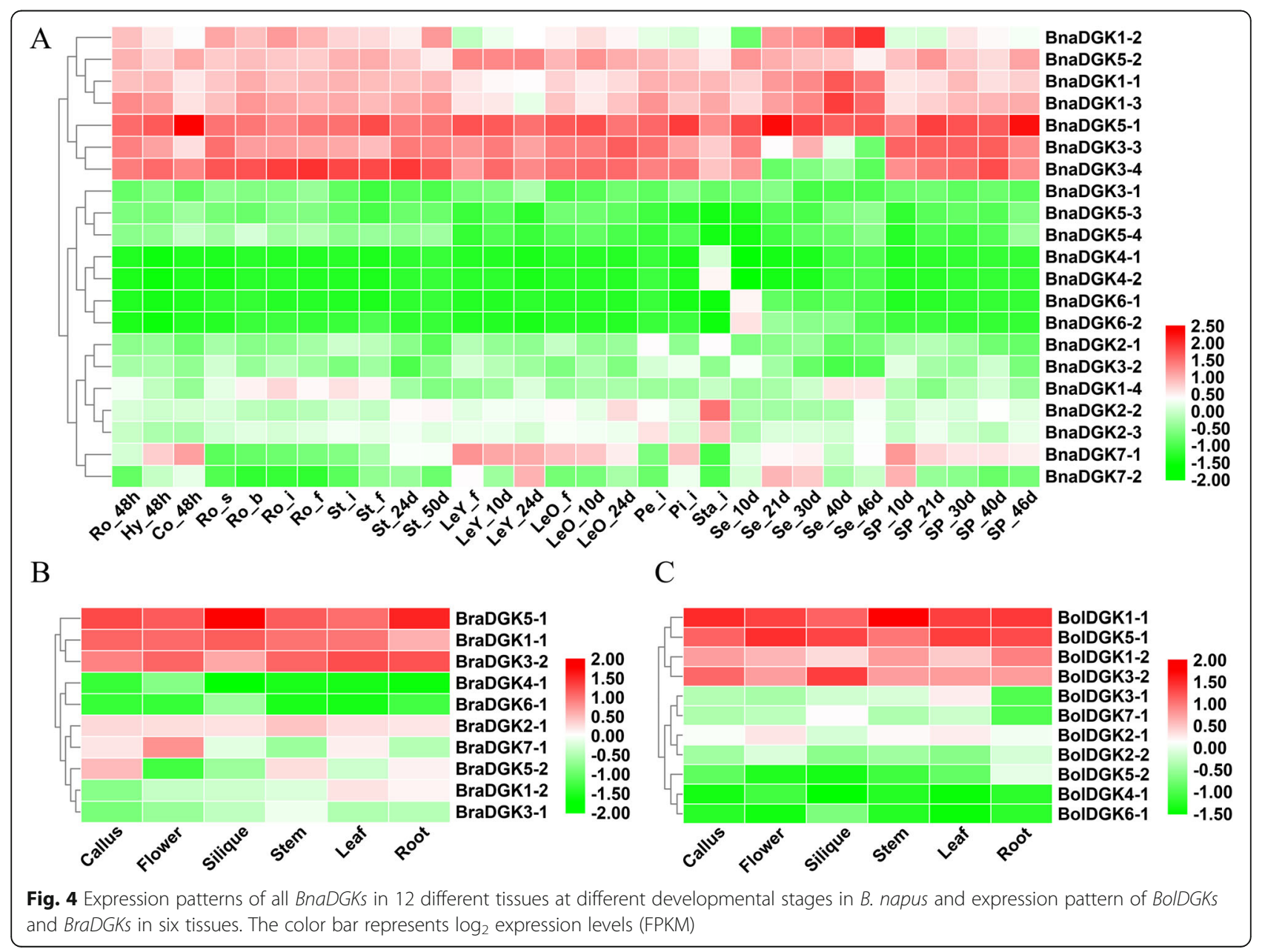


pericarps, respectively. Overall, BnaDGKs had expression detected at different levels in various tissues.

To further study the function of $D G K s$, we then analyzed the expression levels of $D G K s$ in six tissues of parental lines B. rapa and B. oleracea (Fig. $4 \mathrm{~b}$, and c). We also observed that the orthologs members of DGKs exhibited different tissue-specific expression patterns. For example, the orthologs of DGK4 and DGK6 in B. rapa and $B$. oleracea displayed the same expression pattern as those of B. napus, which had low or almost no expression in all tissues (Fig. 4). This result suggests that they might be pseudogenes or expressed only at specific developmental stages or under special conditions. However, homologs of DGK1,DGK3, and DGK5 possessed different expression patterns among them. For example, BnaDGK5-1 and BnaDGK5-2 showed markedly higher expression than BnaDGK5-3 and BnaDGK5-4, as did BolDGK5-1 and BraDGK5-1 compared to BolDGK5-2 and $\operatorname{BraDGK5}-2$, respectively. The divergent expression patterns can be explained by the pseudogenization and functional divergence.

\section{Cis-acting elements in the promoters of $D G K$ genes}

To explore the regulatory mechanism of $D G K$ genes, we carried out an analysis of transcription cis-regulatory elements in the 2000-bp regions upstream of DGK gene transcription start sites in three Brassica species. We identified and counted cis-acting elements associated with plant development and growth, abiotic and biotic stresses, phytohormone responses and light responsiveness in the promoters of all DGK genes (Fig. 5). Of 13 cis-acting elements related to plant development and growth, the as- 1 motif (present in $83.3 \%$ of the Brassica $D G K$ promoters) is involved in the root-specific expression, while the GCN4/AACA motif $(28.6 \%)$ is involved in endosperm expression (Additional file 8: Table S3).

We also identified four types of abiotic stress elements in the $D G K$ promoters: defense and stress responsiveness (MYB/MYC/TC-rich repeats), drought responsiveness (MBS and DRE core), low-temperature responsiveness (LTR), and heat stress responsiveness (STRE) (Fig. 5). Apart from this, two cis-elements related to wounding and pathogen response (W box and WUN motif) were also widely distributed in the $D G K$ promoters (Additional file 8: Table S3). For phytohormone-response-related cisacting regulatory elements, all $D G K$ promoters possessed abscisic-acid-responsive element (ABRE). Approximately 83.3 and $71.4 \%$ of the DGK promoters contained methyljasmonate (Meja)-responsive elements (CGTCA motif and TGACG motif) and gibberellin-responsive elements (GARE-motif, TATC-box, and P-box), respectively, and approximately $66.7 \%$ possessed ethylene-responsive element (ERE). Auxin-response elements (AuxRR core and TGA elements) and the salicylic-acid-response element (TCA element) were also present in certain DGK gene promoters (Additional file 8: Table S3). Notably, BraDGK1-1 and BraDGK4-1 had 11 ABREs and 9 EREs in its promoters, respectively. Moreover, light-responsive regulatory elements consisting of 24 types of different elements were predicted in the $D G K$ promoters (Additional file 8: Table S3). These results confirm that the DGK genes play a major role in stress resistance and hormone signaling pathways.

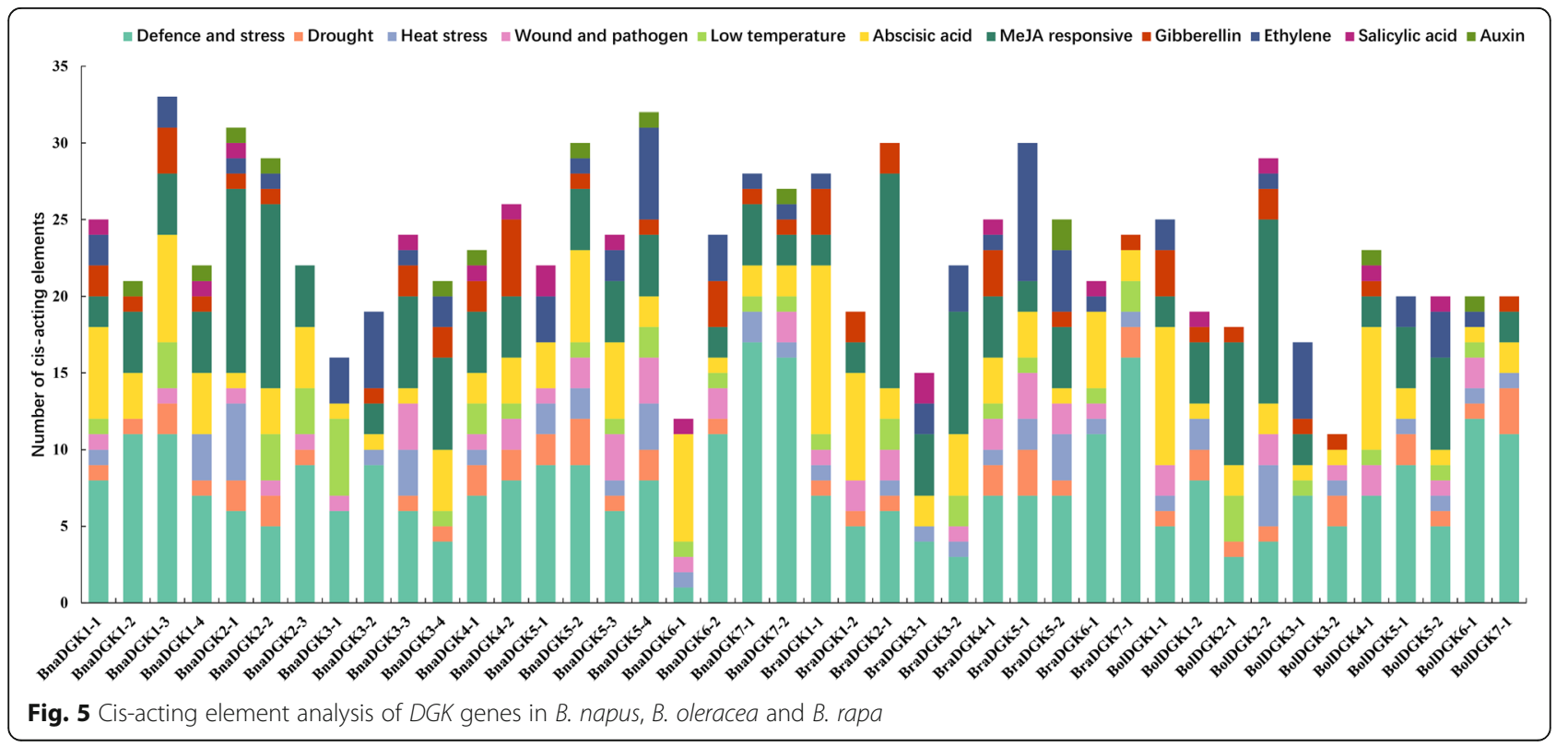


Expression analysis of DGK genes in cultivars with different oil contents

DGAT catalyzes the transfer of an acyl chain from a coenzyme A ester to the sn-3 position of sn-1, 2diacylglycerol to form triacylglycerol [19], while DGKs can catalyze the conversion of DAG to PA. Meanwhile, DGAT and DGK could also compete for the substrate DAG, which is used for the synthesis of TAG and PA, respectively. However, TAG is the main lipid storage form in plants. Therefore, we analyzed the expression patterns of all BnaDGKs in samples from two cultivars with different oil contents using qRT-PCR to explore the relationship between $B n a D G K s$ and seed oil content. The results showed that most BnaDGK genes were differentially expressed in developmental seed and silique pericarp. Except for BnaDGK6-1 had the highest relative expression in 20 DAF seeds, with a change of up to 33 fold, but it had almost no expression in silique pericarp (Fig. 6). In addition, the relative expression of 8 of 21 BnaDGK genes in the seeds, BnaDGK2-2, BnaDGK2-3, BnaDGK3-1, BnaDGK3-2, BnaDGK3-3, BnaDGK3-4, BnaDGK5-2, and BnaDGK7-1, gradually decreased over time (Fig. 6 and Additional file 6: Figure S6). The results suggest that $D G K$ gene expression functions in the early stages of seed development. Importantly, we found that the relative expression levels of BnaDGK2-2, BnaDGK7-1, and BnaDGK7-2 had the higher expression levels in development silique pericarp and seeds of high-oil cultivar than that in the low-oil cultivar, but BnaDGK5-3 and BnaDGK5-4 had conversely expression patterns (Fig. 6), indicating that they might be involved in the accumulation of seed oil contents. Therefore, these DGKS could be selected as excellent candidates for further functional characterization and application in rapeseed breeding programs.

\section{Expression analysis of BnaDGKs under hormone treatment and heavy metal stress}

Based on their expression levels as determined by RNAseq and the closest genetic distance, five gene pairs were selected and their expression patterns were validated under hormone treatments (ABA, BR, and GA) with qRT-PCR. In general, most of DGKs showed a similar expression pattern during the hormone stresses that they showed the sharply up-regulation at $1 \mathrm{~h}$ and decreased to the relatively low expression between $3 \mathrm{~h}$ and $6 \mathrm{~h}$, then their expression levels were gradually increased after $6 \mathrm{~h}$ under ABA and BR treatment (Fig. 7), such as BnaDGK2-2, BnaDGK2-3, and BnaDGK3-3. These results suggested that the gene expression levels of $B n a D G K s$ were obviously induced by exogenous hormone and were affected by their own regulation.

For ABA treatment, expression levels of BnaDGK1-1, BnaDGK2-2, BnaDGK2-3, BnaDGK3-3, and BnaDGK5-2 increased to varying degrees at $1 \mathrm{~h}$, by approximately 2.3-, 5.1-, 4.0-, 2.9-, and 1.5-fold, respectively (Fig. 7). Among these, BnaDGK1-1 and BnaDGK5-2 possess most ABA response cis-elements, whereas BnaDGK2-2 the middle and BnaDGK3-3 the least (Fig. 5). Furthermore, BnaDGK3-4 and BnaDGK5-

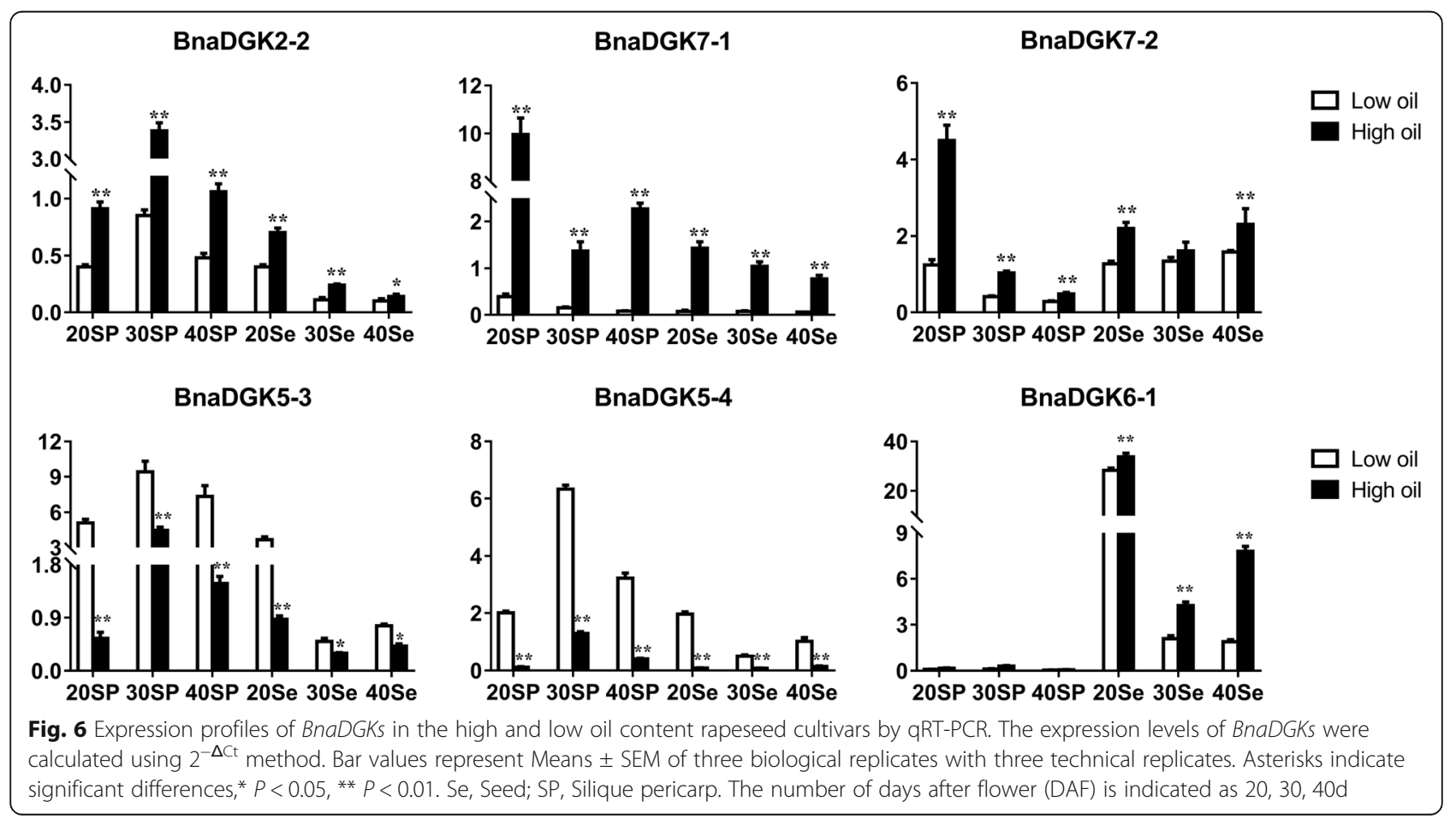



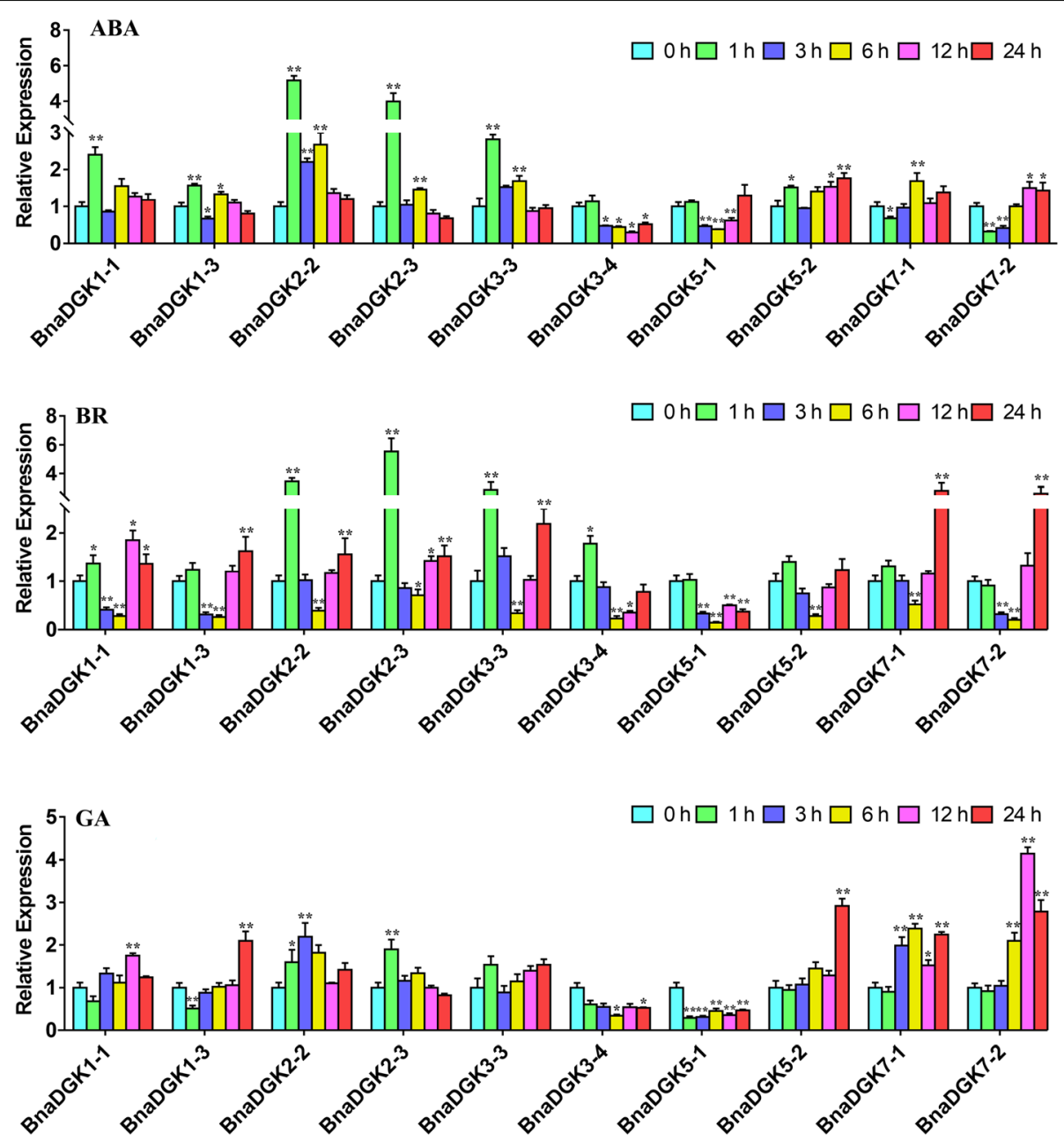

Fig. 7 The expression levels of BnaDGKs in the leaf under hormone treatments by qRT-PCR. A-C, Expression profiles of BnaDGKs under ABA, BR and GA stresses, respectively. The relative expression levels of BnaDGKs were calculated using $2^{-\Delta \Delta C t}$ method and normalized according to the expression values of $\mathrm{O}$ (Control) under hormone treatment. Bar values represent Means \pm SEM of three biological replicates with three technical replicates. Asterisks indicate significant differences, ${ }^{*} P<0.05$, ${ }^{* *} P<0.01$

1 expression profiles were significantly downregulated between $3 \mathrm{~h}$ and $12 \mathrm{~h}$ of ABA exposure, and that of BnaDGK7-2 was initially downregulated and then gradually increased to peak at 12 and $24 \mathrm{~h}$ (Fig. 7). These results suggest that cluster 1 (BnaDGK1 and BnaDGK2) $B n a D G K s$ may be responsive to ABA. We only found that the expression pattern of BnaDGK1 under the ABA treatment was perhaps correlated with the cis-element accumulation (Fig. 5). In terms of BR induction, most $B n a D G K$ genes displayed a similar expression pattern. As BR treatment continued, BnaDGK expression levels decreased between $1 \mathrm{~h}$ and $6 \mathrm{~h}$, and then increased from $6 \mathrm{~h}$ to $24 \mathrm{~h}$. In particular, we observed that the expression levels of BnaDGK2-2, BnaDGK2-3, and $B n a D G K 3-3$ obviously increased at $1 \mathrm{~h}$, whereas BnaDGK7-1and BnaDGK7-2 exhibited stronger increase at $24 \mathrm{~h}$. The expression levels of most BnaDGKs were higher at $24 \mathrm{~h}$ than at $0 \mathrm{~h}$, indicating that the BR response mechanism is complex and the role of $D G K$ genes is minimal and delayed (Fig. 7). The GA treatment results revealed that except for BnaDGK1-3, BnaDGK3-4, and BnaDGK5-1, the remaining BnaDGKs were upregulated with various degrees, with BnaDGK52 and BnaDGK7-2 exhibiting 2.8-, and 4.0-fold upregulation, respectively. After the GA application, BnaDGK1-3 expression gradually increased, peaking at $24 \mathrm{~h}$. In addition, BnaDGK3-4 and BnaDGK5-1 were downregulated by GA treatment. We found in Fig. 7 that BnaDGK5-1 lacked GA cis-elements (Fig. 5). Overall, BnaDGK2-2 and BnaDGK3-3 were upregulated, while BnaDGK3-4 and BnaDGK5-2 were downregulated, in response to the various hormone treatments, implying that some BnaDGK genes respond to hormone induction (Fig. 7).

A previous study reported that $D G K$ genes in land plants can respond to known beneficial elements as well 
as to other metal and metalloid ions [18]. We conducted BnaDGK expression profiling based on RNA-seq data from $B$. napus cultivars subjected to heavy metal induction (Additional file 7: Fig. S7). Upregulation and downregulation were defined by a $\log _{2}$ ratio. The result showed that several BnaDGKs were differentially regulated by $\mathrm{As}^{3+}$ and $\mathrm{Cd}^{2+}$. Thus, induction with $\mathrm{As}^{3+}$ resulted in lower expression of BnaDGK1-4 and BnaDGK3-2, but higher expression of BnaDGK5-4, BnaDGK7-1, and BnaDGK7-2. Application of $30 \mathrm{mg} / \mathrm{L}$ $\mathrm{Cd}^{2+}$ led to the upregulation of BnaDGK1-1, BnaDGK2-2, BnaDGK7-2, and BnaDGK5-4, but downregulation of BnaDGK1-4. Notably, BnaDGK1-4 showed some degree of downregulation in response to all metal ion treatments in all cultivars. Our results suggested that some BnaDGKs might take part in response to heavy metal stress and their expression might be induced by heavy metal treatments in B. napus.

\section{Discussion}

Plants can respond rapidly to environmental stimuli and protect themselves from diverse stressors by activating a series of signal transduction cascades. PA, an emerging signal molecule, mediates signal pathways related to environmental stimuli, and is produced mainly by the PLD and coupled PLC/DGK routes in eukaryotic cells [2]. Moreover, DGK activity has been investigated in several plant species. However, few studies have examined the transcript levels and functions of $D G K$ genes. In this work, we identified 21 BnaDGK genes in B. napus, 10 BraDGK genes in B. rapa, and 11 BolDGK genes in $B$. oleracea. Given that the $A$. thaliana genome encodes 7 $D G K$ genes, it is clear that a WGT event has occurred in Brassica species since they diverged from A. thaliana and that DGK genes have been lost since the WGT event [32]. A report that $35 \%$ of genes were lost via a deletion mechanism after the Arabidopsis and Brassica lineages diverged explains why at least $21 D G K s$ are not observed in either B. rapa or B. oleracea [34]. We identified only 7 collinear gene pairs between $A$. thaliana and $B$. rapa, and 6 collinear gene pairs between $A$. thaliana and $B$. oleracea, perhaps as a result of gene identification or undergo the gene loss during polyploidy speciation. The chromosomal distribution analysis showed that most $D G K$ genes in the Ar subgenome of $B$. rapa and the Co subgenome of $B$. oleracea maintained their positions relative to the $\mathrm{An}$ and $\mathrm{Cn}$ subgenomes of B. napus, respectively. Our synteny analysis between $B$. napus and its diploid progenitors indicated that most $D G K$ genes in Brassica are located in the syntenic regions, with the A subgenome sharing 16 gene pairs and the $C$ subgenome sharing 15 gene pairs.

Polyploidy and WGT are prevalent in the evolutionary history of various species [35]. Most duplicated genes have arisen through whole-genome duplication, and these are often lost or nonfunctional in Brassica species [36]. Exploring the functions and characteristics of duplicate genes is important for understanding plant evolution. Our analysis of the $K_{a} / K_{s}$ values for 69 duplicate genes showed that except for BnaDGK7-2 and BolDGK7-1, all their $K_{a} / K_{s}$ values were $<1$. This implies that these duplicated genes have undergone strong positive selection, which is consistent with previous research indicating that surviving duplicate genes have undergone strong purifying selection [36, 37]. These results indicate that the $D G K$ gene family has been well conserved during evolution over time.

Mammalian DGK enzymes are classified into five groups according to sequence homology, whereas plant $D G K s$ have been divided into three clusters based on analyses in other plant species [38]. The catalytic region of all plant DGKs reported so far consists of a catalytic domain (DGKc, PF00781) followed by an accessory domain (DGKa, PF00609) near the C-terminus, where it may contribute to the normal function of DGKc. The cluster $1 D G K s$ are more complex, with two copies of the DAG-binding domain (C1, PF00130), an upstream basic region, and an extended CRD in their N-termini. The DAG/PE-binding domain features the $\mathrm{C}_{6} / \mathrm{H}_{2}$ cores. By contrast, cluster 2 DGKs harbor only the DGKa and DGKc domains, whereas cluster 3 DGKs may display an extCRD-like domain generated by alternative splicing $[2$, 39]. Moreover, since a GXGXXG consensus sequence in their catalytic domain (DGKc) is the ATP-binding motif, $D G K s$ can use ATP as an energy generator to catalyze the conversion of DAG to PA. Interestingly, DGK1p, a novel DGK gene in the yeast Saccharomyces cerevisiae, utilizes CTP, rather than the standard ATP, as phosphate donor in forming phosphatidate [40]. The sequence of $S$. cerevisiae DGK1p is unlike that of DGKs from other species and contains a CTP transferase domain essential for the protein's $D G K$ activity [40]. The combination of DGK1p and phosphatidate phosphatase encoded by $P A H 1$ can regulate the levels of DAG and PA for phospholipid synthesis [41].

Eukaryotic cells contain two categories of DGKs. On the one hand, inactive $D G K s$, stimulated by inositol phosphate metabolic signaling, can catalyze DAG to generate phosphoglycerols. On the other hand, active $D G K s$ with greater cellular activity can phosphorylate DAG to yield PA [42]. In eukaryotic species, PA is usually produced from the PLD pathway when plants are subjected to drought [43], oxidative stress, or physical damage [16], whereas PA accumulation via the PLC/ DGK pathway is promoted by pathogens and xylanase [16]. Additionally, cold and salt stresses can lead to fast, transient production of PA through both pathways [44]. In $A$. thaliana, transcript expression profiling revealed 
that AtDGK1 and AtDGK2 are implicated in the plant cold response [45]. Furthermore, AtDGK2 expression can be transiently induced by the wounding signal [46]. A recent study showed that $A t D G K 4$ is highly expressed in pollen, and a homozygous AtDGK4 mutation affects not only the male germline but also the vegetative tissue [47]. Overexpression of the rice DGK gene OsBIDK1 can enhance disease resistance in transgenic $N$. tabacum [48], and suppressing OsDGK gene expression results in a distinct depletion of transcripts of the defense gene OsNPR1 and the salt-responsive gene OsCIPK15 [23]. In addition, transcript levels of MdDGK4 and MdDGK8 in apple were induced by salt and drought stress, respectively [4]. In soybean, GmDGK10 transcripts showed dramatic upregulation in response to PEG stress in root tissue, and $G m D G K 8$ and GmDGK9 were significantly upregulated in the presence of $\mathrm{NaCl}$ [24]. Additionally, previous studies have indicated that PA is involved via the PLC/DGK route in various hormone pathways, such as the ethylene [8], ABA [10], and BR [17] pathways. Furthermore, the $D G K$ gene response to beneficial elements and other ions has been demonstrated [18].

Rapeseed is one of the most widely grown oil crops worldwide, and seed oil content is a crucial agronomic trait in rapeseed breeding. Therefore, exploring ways to increase the oil production of $B$. napus is of great agricultural and economic significance. DAG can be catalyzed by DGAT to form TAG, a major contributor to vegetable oils in oilseed crops. Thus we infer that the $D G K$ genes may be candidate genes for improving seed oil content in B. napus. In this study, we investigated the effects of BnaDGK genes on seed oil content using qRTPCR analysis. Several BnaDGK genes were highly expressed in the sample from the high-oil-content cultivar, whereas BnaDGK5-3 and BnaDGK5-4 had distinctly high expression levels in the samples from the low-oil-content cultivar, indicating that differentially expressed BnaDGK gene family members should be further functional identified and applied in rapeseed breeding programs.

Nonetheless, transcription level and functional analysis of the response of $D G K$ gene family members to hormone treatments and metal stress are fragmentary. In terms of hormone treatments, PA interacts with ABI1 phosphatase $2 \mathrm{C}$, thus promoting $\mathrm{ABA}$ signaling in $A$. thaliana [10]. In B. napus root, exogenous application of EBL resulted in PA synthesis through the PLC/DGK pathway under optimal salinity conditions [17]. In our study, cluster 1 BnaDGK genes (BnaDGK1 and BnaDGK2) may respond to ABA treatment. BnaDGK22, BnaDGK3-3, BnaDGK3-4, and BnaDGK5-2 are potential candidate genes in response to hormone treatments, as BnaDGK2-2 and BnaDGK3-3 were upregulated, and BnaDGK3-4 and BnaDGK5-2 were downregulated in response to three types of hormone treatments.

Meanwhile, heavy metals, including copper, manganese, cobalt, zinc, and chromium could be toxic to plants, disrupting plant metabolic processes, inhibiting plant growth, or causing plant death $[49,50]$. Of these, excessive $\mathrm{Cd}$ can produce toxic effects in plants by reducing chlorophyll contents, inhibiting root growth, and perturbing water homeostasis [51]. However, root length, total biomass dry weight, and plant height of $L o$ nicera japonica plants increase in the presence of $0.5-$ $5.0 \mathrm{mg} / \mathrm{L} \mathrm{Cd} \mathrm{[52].} \mathrm{The} \mathrm{expression} \mathrm{levels} \mathrm{of} \mathrm{all} \mathrm{AtDGK}$ genes except AtDGK6 were repressed by $\mathrm{Cd}$, whereas OsDGK8 expression was slightly upregulated [18]. In our study, BnaDGK1-1, BnaDGK2-2, and BnaDGK5-4 were upregulated, while BnaDGK1-4 was downregulated in $B$. napus exposed to $30 \mathrm{mg} / \mathrm{L} \mathrm{Cd}^{2+}$. Our results thus contribute further evidence that $\mathrm{Cd}^{2+}$ influences $D G K$ gene activities.

A previous study has provided direct evidence for As uptake by roots via high-affinity $P H T 1$ transporters in $A$. thaliana and rice [53]. Furthermore, As stimulates the uptake of $\mathrm{Pi}$, providing some growth benefits at the modest concentrations [54]. As (in the form of $\mathrm{NaHAsO} 4)$ upregulates OsDGK2 and downregulates $O s D G K 3 a$ and $O s D G K 8$ expression in rice [18]. In our study, BnaDGK1-4 and BnaDGK3-2 were downregulated, whereas BnaDGK5-4, BnaDGK7-1, and BnaDGK7-2 were upregulated, in response to $\mathrm{As}^{3+}$ treatment. These observations suggest a possible role of $D G K$ genes in $\mathrm{As}^{3+}$ absorption. The cis-acting elements were also predicted from the promoter regions of $D G K$ genes, and a large number of abiotic and biotic stress elements in these promoters were identified, including MYB/MYC/TC-rich repeats, DRE, LTR, and the W box and WUN motif. Apart from these elements, most $D G K$ promotors analyzed contain ABREs, Meja-responsive elements (CGTCA motif and TGACG motif), and gibberellin-responsive elements (GARE-motif, TATCbox, and P-box), as well as EREs.

Together, these results confirm that $D G K$ genes play a major role in stress resistance and hormone signaling pathways. Our findings provide useful information on the evolutionary aspects of the DGK gene family in Brassica genome and assist to reveal the biological functions of DGKs in response to both oil metabolism and abiotic stress.

\section{Conclusions}

In this study, we provided a complete overview of the $D G K$ gene family in B. napus and its diploid progenitors, and analyzed its evolutionary patterns, including gene duplication history and positive selection level. We identified 21, 10, and 11 DGKs in B. napus and its diploid 
progenitors $B$. rapa and B. oleracea, respectively. We also investigated their chromosome distribution, gene structure, domain, and motif pattern, as well as the presence of cis-regulatory elements. The expression patterns of $D G K$ gene family members in diverse tissues at different developmental stages showed that except for those homologous to AtDGK4 and AtDGK6, the DGK genes expressed in most tissues. Further, the expression patterns of $D G K$ genes under hormone treatment and metal ion induction indicated that some $D G K$ genes respond to these treatments. Finally, several BnaDGKs expression profiles in cultivars with different seed oil contents were not completely consistent. Together, these results indicate that $D G K$ genes have various roles in plant growth and development, hormone response, metal ion stress response, and the control of seed oil content. This work improves our understanding of the evolution of the $D G K$ gene family and provides a reference for future studies.

\section{Methods \\ Identification and nomenclature of DGKs in $B$. napus, $B$. rapa and $B$. oleracea}

Genomic sequences, coding sequences, and protein sequences of A. thaliana, B. napus, B. rapa, and B. oleracea were downloaded from the TAIR database (http:// www.arabidopsis.org) and the Brassica Database (BRAD, http://brassicadb.org/brad). Seven AtDGK sequences from $A$. thaliana were used as queries to identify the candidate DGK genes in Brassica species via a BLASTp search with a threshold $e$-value of $1 e-20$ [55]. Furthermore, the Pfam database (http://Pfam.sanger.ac.uk/) and the conserved domain database (CDD) of the US National Center for Biotechnology Information (NCBI) (https://www.ncbi.nlm.nih.gov/Structure/cdd) were used to examine two conserved domains (DGKc, PF00781 and DGKa, PF00609) of the putative DGK genes. In this study, only proteins containing the complete DGKa and DGKc domains were considered as $D G K$ genes.

For nomenclature, a species prefix, such as ' $B n a$ ' for $B$. napus, was used, followed by $D G K$ and two numbers. The first number refers to the homologous Arabidopsis gene, and the second represents the degree of homology to the corresponding Arabidopsis gene, where '-1' represents the highest homology, followed by ' -2 ', and so on: for example BnaDGK1-1.

\section{Multiple sequence alignments and protein properties of} all $D G K$ s in B. napus, B. rapa, B. oleracea, and A. thaliana The multiple sequence alignments of the $D G K$ proteins were established with the ClustalX [56] program, using the default parameter mode. Then, Jalview 2.11.0 [57] and DOG 2.0 [58] software were used to visualize the multiple sequence alignment. In addition, molecular weight $(\mathrm{kDa})$ and isoelectric point $(\mathrm{pI})$ values of each $D G K$ protein sequence were predicted using the ExPASy server (http://expasy.org).

\section{Chromosomal distribution, phylogenetic tree, gene duplication, and syntenic analysis of the DGKs in B. napus, $B$. rapa and $B$. oleracea}

The chromosome location information for the $D G K$ genes of $B$. napus and its two diploid progenitors was collected from the BRAD database, and the chromosome distribution was plotted with MapChart 2.0 [59]. To comprehensively understand the evolutionary relationship of the $D G K$ gene family members, a phylogenetic tree was constructed with MEGA 7.0.26 using the neighbor-joining (NJ) method [60] among four species (A. thaliana, B. napus, B. rapa, and B. oleracea). A series of parameters were as follows: the Poisson model, pairwise deletion, and conserved sequences with coverage of $100 \%$ and 1000 bootstrap replicates. Finally, the phylogenetic tree was visualized using the EvolView website (http://www.evolgenius.info/evolview).

To identify forms of gene duplication of BnaDGKs, only a total of 101,040 B. napus annotated gene sequences were aligned using BLASTp, with an $e$-value of 1e-10. Then, the MCScanX software [61] with default parameters was used to classify the duplication patterns of the DGK genes, and TBtools software [62] was used to calculate the synonymous $\left(K_{s}\right)$ and nonsynonymous $\left(K_{a}\right)$ mutation rates of the duplicated $D G K$ gene pairs. Divergence time was inferred using the formula $\mathrm{T}=K_{s} / 2 \mathrm{R}$, where $K_{s}$ represents the synonymous substitutions per site and $\mathrm{R}$ is the rate of divergence. For dicotyledonous plants, the assumption $\mathrm{R}$ is $1.5 \times 10^{-8}$ synonymous substitutions per site per year [63]. For the syntenic analysis of DGKs, we used Multiple collinear scanning toolkits (MCScanX) to produce the collinearity blocks across the whole genome with the default parameters [61]. Then, the collinearity gene pairs of the $D G K$ family were visualized by Advanced Circos programs of TBtools [64].

Protein domain, motif, and gene structure analysis of all $D G K s$ in B. napus, B. rapa, B. oleracea, and $A$. thaliana

To identify conserved domains of $D G K s$, the NCBI conserved domain search (www.ncbi.nlm.nih.gov/Structure/ cdd/wrpsb.cgi) was used and the results were confirmed by conducting a Pfam database search. A search for conserved motifs of $D G K$ proteins was performed with MEME 5.0.1 (http://meme-suite.org/) using default settings, except that the number of motifs was set to 10 . Subsequently, the Gene Structure Display Server (GSDS 2.0, http://gsds.cbi.pku.edu.cn/) was used to conduct an exon-intron structure analysis of $D G K$ genes. An integrated schematic including the phylogenetic tree, gene 
structure, motif, and conserved domain was visualized using EvolView.

\section{Promoter elements analysis and subcellular localization of} all DGKs in B. napus, B. rapa, B. oleracea, and $A$. thaliana A 2000 bp region upstream of the translation start sites of each $D G K$ gene was acquired from Brassica database $(\mathrm{BRAD})$ as a promoter sequence, and the cis-acting elements were analyzed using the PlantCARE website (http:// bioinformatics.psb.ugent.be/webtools/plantcare/html/). The subcellular localization was predicted using the PSORT online website (http://psort1.hgc.jp/form.html).

\section{Expression analysis of all DGKs in different tissues of $B$. napus, $B$. rapa, and $B$. oleracea}

First, total RNA was isolated using the EZ-10 DNAaway RNA Mini-prep Kit (Sangon Biotech Co., Ltd., Shanghai, China) for the rapeseed cultivar Zhongshuang 11 (ZS11) in distinct tissues at different developmental stages. The cDNA library construction and RNA sequencing were performed as described previously [65] at Novogene Bioinformatics Technology (Beijing, China) and were deposited in the BioProject database (BioProject ID PRJNA358784). Then the relative expression values in the bars were calculated based on FPKM values (fragments per kilobase of exon model per million) using Cufflinks with default parameters [66], and expression patterns of the candidate BnaDGKs were analyzed at 12 tissues (radicle, hypocotyl, cotyledon, root, stem, young leaf, mature leaf, petal, pistil, stamen, seed, and seed pericarp; Additional file 8: Table S4 and Table S5). Furthermore, six tissues (callus, flower, silique, stem, leaf, and root) RNA-seq data of cultivated B. oleracea and $B$. rapa were obtained from the NCBI GEO database (https://www.ncbi.nlm.nih.gov/geo/browse/; accession numbers GSE42891 and GSE43245). All BnaDGKs, BraDGKs, and BolDGKs expression levels were also estimated using FPKM (Additional file 8: Table S5 and Table S6).

In addition, transcriptome sequencing datasets of five rapeseed cultivars under heavy metal stress were generated and analyzed as described above, which were treated cultivars P070, P085, and P087 with $15 \mathrm{mg} / \mathrm{L}$ $\mathrm{As}^{3+}$ and cultivars P085, P155, and P163 with $30 \mathrm{mg} / \mathrm{L}$ $\mathrm{Cd}^{2+}$, respectively (Additional file 8: Table S7). Gene expression levels of BnaDGKs were estimated using FPKM values. Heatmaps of all DGKs were drafted using TBtools [64], which was normalized by $\log _{2}$ (FPKM values).

Plant materials, hormone treatments, and heavy metal stress

Two B. napus cultivars were used in this study, P594 (high oil content, 43\%) and P134 (low oil content, 34\%), both provided by the Chongqing Rapeseed Engineering Technology Research Center. Both cultivars were grown under natural conditions in Chongqing, China, and inflorescences were bagged before blossoming to prevent pollen contamination. Finally, developing seeds and silique pericarp of two cultivars were collected at 20, 30, and 40 days after flowering (DAF) and preserved at $80^{\circ} \mathrm{C}$ for further analysis.

For hormone treatment, ZS11 seeds were sown and grown in an artificial climatic chamber at $25^{\circ} \mathrm{C}$ with a $16 / 8 \mathrm{~h}$ (day/night) photoperiod. Two-week-old ZS11 seedlings were treated with $50 \mu \mathrm{M}$ ABA, GA, or BR for $0,1,3,6,12$, and $24 \mathrm{~h}$, respectively, and their leaves were immediately collected. All materials were frozen in liquid nitrogen and stored at $-80^{\circ} \mathrm{C}$ until use.

Five rapeseed cultivars (P070, P085, P087, P155, and P163) with extreme phenotypes for heavy metal response (strong of weak resistance) were selected from two hundred rapeseed accessions under different concentrations of two heavy metals $\left(\mathrm{As}^{3+}\right.$ and $\left.\mathrm{Cd}^{2+}\right)$. Subsequently, we treated rapeseed cultivars (P070, P085, and P087) with $15 \mathrm{mg} / \mathrm{L} \mathrm{As}^{3+}$ and rapeseed cultivars (P085, P155, and P163) with $30 \mathrm{mg} / \mathrm{L} \mathrm{Cd}^{2+}$ as the optimal concentration in this study. In brief, the filled seeds for each accession were sown on the filter papers in the petri dishes $(9 \times 9$ $\mathrm{cm})$, which were treated with distilled water (Control) and $15 \mathrm{mg} / \mathrm{L} \mathrm{As}^{3+}$ and $30 \mathrm{mg} / \mathrm{L} \mathrm{Cd}^{2+}$ (Treatment), respectively. The growth conditions were kept at $25^{\circ} \mathrm{C}$ with long-day conditions ( $16 \mathrm{~h}$ light $/ 8 \mathrm{~h}$ dark, $5000 \mathrm{Lux}$ ). After 7 days, whole plants were frozen in liquid nitrogen and stored at $-80^{\circ} \mathrm{C}$ until use.

\section{RNA extraction, complementary DNA synthesis, and quantitative real-time PCR analysis of BnaDGK genes} Total RNA was extracted with the EZ-10 DNAaway RNA Mini-prep Kit (Sangon Biotech Co., Ltd., Shanghai, China) according to the manufacturer's instructions. Subsequently, the gel electrophoresis and a NanoDrop 2000 spectrophotometer were used for detecting the quality and concentration of each RNA sample, and the qualified RNA were used for further analysis. The firststrand cDNA was synthesized from $1 \mu \mathrm{g}$ RNA by reverse transcription PCR (RT-PCR) with a Perfect Real-Time Synthesis Kit (TaKaRa Biotechnology, Dalian, China). The diluted cDNA after reverse transcription was used as the template for real-time quantitative RT-PCR. Realtime quantitative PCR was conducted with a BIO-RAD CFX96 qPCR instrument and SYBR II (TaKaRa). Each $20 \mu \mathrm{l} \mathrm{PCR}$ mixture that included $10 \mu \mathrm{l}$ of SYBR ${ }^{\circ}$ Premix $\mathrm{Ex} \mathrm{Taq}^{\mathrm{ma}}$ II, $2 \mu \mathrm{l}(100 \mathrm{ng})$ of template cDNA, and $0.4 \mu \mathrm{M}$ of each PCR primer. The RT-PCR protocol was set to $95^{\circ} \mathrm{C}$ for $30 \mathrm{~s}$ and 35 cycles of $95^{\circ} \mathrm{C}$ for $5 \mathrm{~s}$, followed by $56-60{ }^{\circ} \mathrm{C}$ (depending on the primers used) for $30 \mathrm{~s}$. All 
samples were amplified in three biological replicates and three technical replicates. The $2^{-\Delta \Delta \mathrm{Ct}}$ value was used to measure the relative expression levels of $B n D G K s$ under hormone treatment [67]; and the $2^{-\Delta \mathrm{Ct}}$ value was used to measure the relative expression levels of $B n D G K s$ between low and high oil content rapeseed [68]. B. napus ACTIN7 (BnaACTIN7, GenBank Accession No. AF024716) was used as the housekeeping gene. All qRTPCR primers are listed in Additional file 8: Table S8.

\section{Statistical analysis}

The expression levels were defined as mean \pm standard error of mean (SEM), and three biological replicates were performed in each experiment. Data was subjected to a one-way ANOVA using SPSS 15.0 (SPSS, Inc., Chicago, Ill) to define the significance differences between the mean values. Differences with $p$ values of $\leq 0.05$ and $\leq 0.01$ were considered significant and highly significant, respectively.

\section{Supplementary information}

Supplementary information accompanies this paper at https://doi.org/10. 1186/s12870-020-02691-y.

Additional file 1: Figure S1. Chromosomal distribution of DGKs in $B$. napus, B. rapa and B. oleracea, the scale bar is showed in the figure.

Additional file 2: Figure S2. The multiple sequence alignment of two DAG/PE-binding domain (C1 domain) domains. (A) The first DAG/PEbinding domain, (B) The second DAG/PE-binding domain.

Additional file 3: Figure S3. The multiple sequence alignment of each cluster DGKc domain among all DGK genes.

Additional file 4: Figure S4. A detailed view of domains of DGK genes in cluster 1 including two $\mathrm{C} 1$ domains, the upstream basic regions and the extended cysteine-rich domain (extCRD).

Additional file 5: Figure S5. The detailed information of Motif logos are obtained from the MEME Suite website.

Additional file 6: Figure S6. Quantitative RT-PCR analysis of the remaining BnaDGKs in different seed oil content materials. The expression levels of BnaDGKs were calculated using $2^{-\Delta C t}$ method. Bar values represent Means \pm SEM of three biological replicates with three technical replicates. Asterisks indicate significant differences, ${ }^{*} P<0.05,{ }^{* *} P<0.01$. Se, Seed; SP, Silique pericarp. The number of days after flower (DAF) is indicated as 20, 30, 40d.

Additional file 7: Figure S7. The heatmap of BnaDGKs expression profiling in response to $\mathrm{As}$ and $\mathrm{Cd}$ stress. The up-down regulation was defined with $\log _{2}$ ratio.

Additional file 8: Table S1. Identification of DGKs in B. napus and its diploid progenitors with their physico-chemical and bio-chemical properties (ER: Endoplasmic reticulum; Nucl: Nucleus; Pero: Peroxisome; Mito: Mitochondria; Chlo: Chloroplast; Cyto: Cytoplasm); Table S2. The $K_{a} / K_{s}$ values and MYA for duplicated DGK gene pairs; Table S3. The detailed information of cis-acting analysis among DGKs in B. napus, B. oleracea, and B. rapa; Table S4. B. napus ZS11 tissues and organs in this study; Table S5. The FPKM values of DGK genes in B. napus by RNA-Seq anaIysis; Table S6. The FPKM values of DGK genes in B. rapa and B. oleracea by RNA-Seq analysis; Table S7. The FPKM values of DGK genes in $B$. napus by RNA-Seq analysis under metal ion stress; Table S8. Primers used to amplify the BnaDGK genes and reference genes using qRT-PCR.

\section{Abbreviations}

DGK: Diacylglycerol kinase; DAG: Diacylglycerol; PA: Phosphatidic acid; $D G K c$ : Diacylglycerol kinase catalytic; DGKa: Diacylglycerol kinase accessory; WGT: Whole-genome triplication; QRT-PCR: Quantitative real-time polymerase chain reaction; $A B A$ : Abscisic acid; $B R$ : Brassinolide; GA: Gibberellic acid; PLC: Phospholipase C; PLD: Phospholipase D; PC: Phosphatidylcholine; EBL: 24-epibrassinolide; As: Arsenic; Cd: Cadmium; DGAT: Diacylglycerol transferase; TAG: Triacylglycerol; DAG/PE: Diacylglycerol/phorbol ester; Ka: Nonsynonymous; Ks: Synonymous; MYA: Million years ago; ABRE: Abscisicacid-responsive element; ERE: Ethylene-responsive element; DAF: Days after flowering; NJ: Neighbor-joining; FPKM: Framents per kilobase of exon per million mapped framents

\section{Acknowledgements}

Not applicable.

\section{Authors' contributions}

CMQ and JNL conceived and guided the project. FT and ZCX conducted the experiments. FJS, SLS, and SC collected and analyzed the data; RC, MCZ, and QWZ carried out the experiments and performed software; $\mathrm{HD}$ and $\mathrm{KL}$ analyzed the transcriptome data; $\mathrm{FT}, \mathrm{KL}$, and CMQ wrote the manuscript; JNL and $\mathrm{CMQ}$ reviewed the manuscript. All authors have read and approved the final manuscript.

\section{Funding}

This research was funded by National Key R\&D Program of China (2018YFD0100500), the National Natural Science Foundation of China (31830067), Fundamental Research Funds for the Central Universities (XDJK2020B030, XDJK2019C099), and the "111" Project (B12006), Modern Agro-industry Technology Research System (CARS-13).

\section{Availability of data and materials}

RNA-seq of B. napus variety Zhongshuang 11 (ZS11) in distinct tissues at different developmental stages are available in the NCBI Sequence Read Archive (SRA) database under the accession number PRJNA358784. RNA-seq data for expression profiles of $B$. rapa and B. oleracea in different tissues were acquired from NCBI gene expression omnibus (GEO) database (GSE43245 and GSE42891). All other datasets supporting the results of this article are included within the article and its Additional files.

Ethics approval and consent to participate

Not applicable.

\section{Consent for publication}

Not applicable.

\section{Competing interests}

The authors declare that they have no competing interests.

\section{Author details}

${ }^{1}$ Chongqing Engineering Research Center for Rapeseed, College of Agronomy and Biotechnology, Southwest University, Chongqing 400716, China. ${ }^{2}$ Academy of Agricultural Sciences, Southwest University, Chongqing 400715, China.

Received: 1 May 2020 Accepted: 8 October 2020 Published online: 15 October 2020

\section{References}

1. Hou QC, Ufer GD, Bartels D. Lipid signalling in plant responses to abiotic stress. Plant Cell Environ. 2016;39(5):1029-48.

2. Arisz SA, Testerink C, Munnik T. Plant PA signaling via diacylglycerol kinase. BBA-Mol Cell Biol L. 2009;1791(9):869-75.

3. Arisz SA, van Himbergen JAJ, Musgrave A, van den Ende H, Munnik T. Polar glycerolipids of Chlamydomonas moewusii. Phytochemistry. 2000; 53(2):265-70.

4. Li YL, Tan YX, Shao Y, Li MJ, Ma FW. Comprehensive genomic analysis and expression profiling of diacylglycerol kinase gene family in Malus prunifolia (Willd.) Borkh. Gene. 2015;561(2):225-34. 
5. Zhang Q, Lin F, Mao TL, Nie JN, Yan M, Yuan M, Zhang WH. Phosphatidic acid regulates microtubule organization by interacting with MAP 65-1 in response to salt stress in Arabidopsis. Plant Cell. 2012;24(11):4555-76.

6. Arisz SA, van Wijk R, Roels W, Zhu JK, Haring MA, Munnik T. Rapid phosphatidic acid accumulation in response to low temperature stress in Arabidopsis is generated through diacylglycerol kinase. Front Plant Sci. 2013;4:1.

7. Munnik T, Meijer HJG, ter Riet B, Hirt H, Frank W, Bartels D, Musgrave A. Hyperosmotic stress stimulates phospholipase D activity and elevates the levels of phosphatidic acid and diacylglycerol pyrophosphate. Plant J. 2000; 22(2):147-54.

8. Munnik T. Phosphatidic acid: an emerging plant lipid second messenger. Trends Plant Sci. 2001;6(5):227-33.

9. Zhang Q, Xiao SY. Lipids in salicylic acid-mediated defense in plants: focusing on the roles of phosphatidic acid and phosphatidylinositol 4phosphate. Front Plant Sci. 2015;6:387.

10. Zhang W, Qin C, Zhao J, Wang X. Phospholipase Da1-derived phosphatidic acid interacts with $\mathrm{ABI} 1$ phosphatase $2 \mathrm{C}$ and regulates abscisic acid signaling. Proc Natl Acad Sci U S A. 2004;101(25):9508-13.

11. Derevyanchuk M, Kretynin S, Kolesnikov Y, Litvinovskaya R, Martinec J, Khripach $V$, Kravets $V$. Seed germination, respiratory processes and phosphatidic acid accumulation in Arabidopsis diacylglycerol kinase knockouts - the effect of brassinosteroid, brassinazole and salinity. Steroids. 2019;147:28-36

12. Villasuso AL, Di Palma MA, Aveldano M, Pasquare SJ, Racagni G, Giusto NM, Machado EE. Differences in phosphatidic acid signalling and metabolism between $A B A$ and GA treatments of barley aleurone cells. Plant Physiol Bioch. 2013;65:1-8

13. Villasuso AL, Molas ML, Racagni G, Abdala G, Machado-Domenech E. Gibberellin signal in barley aleurone: early activation of PLC by $G$ protein mediates amylase secretion. Plant Growth Regul. 2003;41(3):197-205.

14. Li MY, Hong YY, Wang XM. Phospholipase D- and phosphatidic acidmediated signaling in plants. BBA-Mol Cell Biol L. 2009;1791(9):927-35.

15. Testerink C, Munnik T. Molecular, cellular, and physiological responses to phosphatidic acid formation in plants. J Exp Bot. 2011;62(7):2349-61.

16. Testerink C, Munnik T. Phosphatidic acid: a multifunctional stress signaling lipid in plants. Trends Plant Sci. 2005;10(8):368-75.

17. Derevyanchuk M, Kretynin S, lakovenko O, Litvinovskaya $R$, Zhabinskii $V$, Martinec J, Blume Y, Khripach V, Kravets V. Effect of 24-epibrassinolide on Brassica napus alternative respiratory pathway, guard cells movements and phospholipid signaling under salt stress. Steroids. 2017;117:16-24.

18. Escobar-Sepulveda HF, Trejo-Tellez LI, Perez-Rodriguez P, Hidalgo-Contreras $J$, Gomez-Merino FC. Diacylglycerol kinases are widespread in higher plants and display inducible gene expression in response to beneficial elements, metal, and metalloid ions. Front Plant Sci. 2017:8:129.

19. Bates $P$, Browse J. The significance of different diacylgycerol synthesis pathways on plant oil composition and bioengineering. Front Plant Sci. 2012;3:147.

20. Snedden WA, Blumwald E. Alternative splicing of a novel diacylglycerol kinase in tomato leads to a calmodulin-binding isoform. Plant J. 2000;24(3): 317-26.

21. Gomez-Merino FC, Brearley CA, Ornatowska M, Abdel-Haliem MEF, Zanor MI, Mueller-Roeber B. AtDGK2, a novel diacylglycerol kinase from Arabidopsis thaliana, phosphorylates 1-stearoyl-2-arachidonoyl-sn-glycerol and 1,2dioleoyl-sn-glycerol and exhibits cold-inducible gene expression. J Bio Chem. 2004;279(9):8230-41.

22. Gu YN, Zhao CJ, He L, Yan BW, Dong JJ, Li ZT, Yang KJ, Xu JY. Genome-wide identification and abiotic stress responses of DGK gene family in maize. J Plant Biochem Biot. 2018;27(2):156-66.

23. Ge HL, Chen C, Jing W, Zhang Q, Wang H, Wang R, Zhang WH. The rice diacylglycerol kinase family: functional analysis using transient RNA interference. Front Plant Sci. 2012:3:60.

24. Carther KFI, Ketehouli T, Ye N, Yang YH, Wang N, Dong YY, Yao N, Liu XM, Liu WC, Li XW, et al. Comprehensive genomic analysis and expression profiling of Diacylglycerol kinase (DGK) gene family in soybean (Glycine max) under abiotic stresses. Int J Mol Sci. 2019;20(6):1361.

25. Chalhoub B, Denoeud F, Liu SY, Parkin IAP, Tang HB, Wang XY, Chiquet J, Belcram H, Tong CB, Samans B, et al. Early allopolyploid evolution in the post-Neolithic Brassica napus oilseed genome. Science. 2014; 345(6199):950-3.
26. Wang XW, Wang HZ, Wang J, Sun RF, Wu J, Liu SY, Bai YQ, Mun JH, Bancroft I, Cheng F, et al. The genome of the mesopolyploid crop species Brassica rapa. Nat Genet. 2011;43(10):1035-U1157.

27. Liu SY, Liu YM, Yang XH, Tong CB, Edwards D, Parkin IAP, Zhao MX, Ma JX, Yu JY, Huang SM, et al. The Brassica oleracea genome reveals the asymmetrical evolution of polyploid genomes. Nat Commun. 2014;5:3930.

28. Lundberg GA, Sommarin M. Diacylglycerol kinase in plasma-membranes from wheat. Biochim Biophys Acta. 1992;1123(2):177-83.

29. Hendrix KW, Assefa H, Boss WF. The polyphosphoinositides, phosphatidylinositol monophosphate and phosphatidylinositol bisphosphate, are present in nuclei isolated from carrot protoplast. Protoplasma. 1989;151(1):62-72.

30. Vaultier M-N, Cantrel C, Guerbette F, Boutte Y, Vergnolle C, Cicek D, Bolte S, Zachowski A, Ruelland E. The hydrophobic segment of Arabidopsis thaliana cluster I diacylglycerol kinases is sufficient to target the proteins to cell membranes. FEBS Lett. 2008;582(12):1743-8.

31. Pears CJ, Kour G, House C, Kemp BE, Parker PJ. Mutagenesis of the pseudosubstrate site of protein kinase C leads to activation. Eur J Biochem. 1990;194(1):89-94.

32. Cheng F, Wu J, Wang XW. Genome triplication drove the diversification of Brassica plants. Hortic Res-England. 2014;1:14014.

33. Azzi A, Boscoboinik D, Hensey C. The protein kinase C family. Eur J Biochem. 1992;208(3):547-57.

34. Town CD, Cheung F, Maiti R, Crabtree J, Haas BJ, Wortman JR, Hine EE, Althoff R, Arbogast TS, Tallon LJ, et al. Comparative genomics of Brassica oleracea and Arabidopsis thaliana reveal gene loss, fragmentation, and dispersal after polyploidy. Plant Cell. 2006;18(6):1348-59.

35. Panchy N, Lehti-Shiu M, Shiu SH. Evolution of gene duplication in plants. Plant Physiol. 2016;171(4):2294-316.

36. Zou C, Lehti-Shiu MD, Thomashow M, Shiu SH. Evolution of stress-regulated gene expression in duplicate genes of Arabidopsis thaliana. PLoS Genet. 2009;5(7):e1000581.

37. Lynch M, Conery JS. The evolutionary fate and consequences of duplicate genes. Science. 2000;290(5494):1151-5.

38. Sakane F, Imai S, Kai M, Yasuda S, Kanoh H. Diacylglycerol kinases: why so many of them? BBA-Mol Cell Biol L. 2007;1771(7):793-806.

39. Cacas JL, Gerbeau-Pissot P, Fromentin J, Cantrel C, Thomas D, Jeannette E, Kalachova T, Mongrand S, Simon-Plas F, Ruelland E. Diacylglycerol kinases activate tobacco NADPH oxidase-dependent oxidative burst in response to cryptogein. Plant Cell Environ. 2017:40(4):585-98.

40. Han GS, O'Hara L, Carman GM, Siniossoglou S. An unconventional diacylglycerol kinase that regulates phospholipid synthesis and nuclear membrane growth. J of Bio Chem. 2008;283(29):20433-42.

41. Qiu YX, Fakas S, Han GS, Barbosa AD, Siniossoglou S, Carman GM. Transcription factor Reb1p regulates DGK1-encoded Diacylglycerol kinase and lipid metabolism in Saccharomyces cerevisiae. J of Bio Chem. 2013; 288(40):29124-33

42. Wang X, Su Y, Liu Y, Kim S-C, Fanella B. Phosphatidic acid as lipid messenger and growth regulators in plants. In: Wang $X$, editor. Phospholipases in Plant Signaling, vol. 20; 2014. p. 69-92.

43. Hong YY, Zheng SQ, Wang XM. Dual functions of phospholipase D alpha 1 in plant response to drought. Mol Plant. 2008;1(2):262-9.

44. Ruelland E, Cantrel C, Gawer M, Kader JC, Zachowski A. Activation of phospholipases $C$ and $D$ is an early response to a cold exposure in Arabidopsis suspension cells. Plant Physiol. 2002;130(2):999-1007.

45. Lee BH, Henderson DA, Zhu JK. The Arabidopsis cold-responsive transcriptome and its regulation by ICE1. Plant Cell. 2005;17(11):3155-75.

46. Gomez-Merino FC, Arana-Ceballos FA, Trejo-Tellez LI, Skirycz A, Brearley CA, Dormann P, Mueller-Roeber B. Arabidopsis AtDGK7, the smallest member of plant diacylglycerol kinases (DGKs), displays unique biochemical features and saturates at low substrate concentration - the DGK inhibitor R59022 differentially affects AtDGK2 and AtDGK7 activity in vitro and alters plant growth and development. J of Bio Chem. 2005;280(41):34888-99.

47. Dias FV, Serrazina S, Vitorino M, Marchese D, Heilmann I, Godinho M, Rodrigues M, Malho R. A role for diacylglycerol kinase 4 in signalling crosstalk during Arabidopsis pollen tube growth. New Phytol. 2019;222(3): 1434-46.

48. Zhang WD, Chen J, Zhang HJ, Song FM. Overexpression of a rice diacylglycerol kinase gene OsBIDK1 enhances disease resistance in transgenic tobacco. Mol Cells. 2008;26(3):258-64. 
49. Ebbs S, Uchil S. Cadmium and zinc induced chlorosis in Indian mustard [Brassica juncea (L.) Czern] involves preferential loss of chlorophyll b. Photosynthetica. 2008;46(1):49-55.

50. Lingua G, Franchin C, Todeschini V, Castiglione S, Biondi S, Burlando B, Parravicini V, Torrigiani P, Berta G. Arbuscular mycorrhizal fungi differentially affect the response to high zinc concentrations of two registered poplar clones. Environ Pollut. 2008;153(1):137-47.

51. Chen X, Wang J, Shi Y, Zhao MQ, Chi GY. Effects of cadmium on growth and photosynthetic activities in pakchoi and mustard. Bot Stud. 2011;52(1):41-6.

52. Liu ZL, Chen W, He XY, Jia L, Yu S, Zhao MZ. Hormetic responses of Lonicera Japonica Thunb. To Cadmium Stress. Dose-Response. 2015;13(1):14-33.

53. Li NN, Wang JC, Song WY. Arsenic uptake and translocation in plants. Plant Cell Physiol. 2016;57(1):4-13.

54. Tu S, Ma LQ. Interactive effects of $\mathrm{pH}$, arsenic and phosphorus on uptake of As and $P$ and growth of the arsenic hyperaccumulator Pteris vittata $\mathrm{L}$. under hydroponic conditions. Environ Exp Bot. 2003;50(3):243-51.

55. Altschul SF, Madden TL, Schaffer AA, Zhang J, Zhang Z, Miller W, Lipman DJ. Gapped BLAST and PSI-BLAST: a new generation of protein database search programs. Nucleic Acids Res. 1997;25(17):3389-402.

56. Thompson JD, Gibson TJ, Plewniak F, Jeanmougin F, Higgins DG. The CLUSTAL_X windows interface: flexible strategies for multiple sequence alignment aided by quality analysis tools. Nucleic Acids Res. 1997;25(24): 4876-82.

57. Waterhouse AM, Procter JB, Martin DM, Clamp M, Barton GJ. Jalview version 2--a multiple sequence alignment editor and analysis workbench. Bioinformatics. 2009;25(9):1189-91.

58. Ren J, Wen L, Gao X, Jin C, Xue Y, Yao X. DOG 1.0: illustrator of protein domain structures. Cell Res. 2009;19(2):271-3.

59. Voorrips RE. MapChart: software for the graphical presentation of linkage maps and QTLs. J Hered. 2002;93(1):77-8.

60. Kumar S, Stecher G, Tamura K. MEGA7. Molecular evolutionary genetics analysis version 7.0 for bigger datasets. Mol Biol Evol. 2016;33(7):1870-4.

61. Wang Y, Tang H, Debarry JD, Tan X, Li J, Wang X, Lee TH, Jin H, Marler B, Guo H, et al. MCScanX: a toolkit for detection and evolutionary analysis of gene synteny and collinearity. Nucleic Acids Res. 2012;40(7):e49.

62. Chen C, Chen H, He Y, Xia R. TBtools, a toolkit for biologists integrating various biological data handling tools with a user-friendly interface. BioRxiv. 2018. p. 289660.

63. Koch MA, Haubold B, Mitchell-Olds T. Comparative evolutionary analysis of chalcone synthase and alcohol dehydrogenase loci in Arabidopsis, Arabis, and related genera (Brassicaceae). Mol Biol Evol. 2000;17(10):1483-98.

64. Chen C, Chen H, Zhang Y, Thomas HR, Frank MH, He Y, Xia R. TBtools: an integrative toolkit developed for interactive analyses of big biological data. Mol Plant. 2020. https://doi.org/10.1016/j.molp.2020.06.009.

65. Qu C, Fu F, Liu M, Zhao H, Liu C, Li J, Tang Z, Xu X, Qiu X, Wang R, et al. Comparative Transcriptome analysis of recessive male sterility (RGMS) in sterile and fertile Brassica napus lines. PLoS One. 2015;10(12):e0144118.

66. Trapnell C, Roberts A, Goff L, Pertea G, Kim D, Kelley DR, Pimentel H, Salzberg SL, Rinn JL, Pachter L. Differential gene and transcript expression analysis of RNA-seq experiments with TopHat and cufflinks. Nat Protoc. 2012;7(3):562-78

67. Livak KJ, Schmittgen TD. Analysis of relative gene expression data using real-time quantitative PCR and the $2^{-\Delta \Delta C T}$ method. Methods. 2001;25(4): 402-8.

68. Sheng X, Zhao Z, Wang J, Yu H, Shen Y, Zeng X, Gu H. Genome wide analysis of MADS-box gene family in Brassica oleracea reveals conservation and variation in flower development. BMC Plant Biol. 2019;19:106.

\section{Publisher's Note}

Springer Nature remains neutral with regard to jurisdictional claims in published maps and institutional affiliations.

Ready to submit your research? Choose BMC and benefit from:

- fast, convenient online submission

- thorough peer review by experienced researchers in your field

- rapid publication on acceptance

- support for research data, including large and complex data types

- gold Open Access which fosters wider collaboration and increased citations

- maximum visibility for your research: over $100 \mathrm{M}$ website views per year

At BMC, research is always in progress.

Learn more biomedcentral.com/submissions 